Revue des patrimoines

\title{
Un patrimoine redécouvert : les dépôts du Centre national des arts plastiques à l'École nationale supérieure des beaux-arts de Paris (1809-1923)
}

A heritage rediscovered, the deposits of the Centre national des arts plastiques at

the Paris École des Beaux-Arts, 1809-1923

\section{Stéphane Allavena}

\section{OpenEdition}

Journals

Édition électronique

URL : http://journals.openedition.org/insitu/21013

DOI : 10.4000/insitu.21013

ISSN : $1630-7305$

Éditeur

Ministère de la Culture

Référence électronique

Stéphane Allavena, «Un patrimoine redécouvert : les dépôts du Centre national des arts plastiques à l'École nationale supérieure des beaux-arts de Paris (1809-1923) », In Situ [En ligne], 38 | 2019, mis en ligne le 15 février 2019, consulté le 10 décembre 2020. URL : http://journals.openedition.org/insitu/ 21013 ; DOI : https://doi.org/10.4000/insitu.21013

Ce document a été généré automatiquement le 10 décembre 2020.

\section{c) $(1) \odot$}

In Situ Revues des patrimoines est mis à disposition selon les termes de la licence Creative Commons Attribution - Pas d'Utilisation Commerciale - Pas de Modification 4.0 International. 


\title{
Un patrimoine redécouvert : les dépôts du Centre national des arts plastiques à l'École nationale supérieure des beaux-arts de Paris (1809-1923)
}

\author{
A heritage rediscovered, the deposits of the Centre national des arts plastiques at \\ the Paris École des Beaux-Arts, 1809-1923
}

\section{Stéphane Allavena}

Installée depuis 1829 sur le site de l'ancien musée des Monuments français ouvert au public par Alexandre Lenoir en 1795, l'École nationale supérieure des beaux-arts abrite une collection riche de plus de 450000 objets, bénéficiaire par arrêté du 10 juillet 2017 du label «Musée de France». Composée de travaux d'élèves issus des différents concours organisés en son sein ainsi que de nombreux dons et legs, elle accueille également un important dépôt d'œuvres d'art, propriété du Centre national des arts plastiques (CNAP). Ce dernier, héritier de l'un des services de la division des BeauxArts, des Sciences et des Spectacles, créée en 1791 pour soutenir la création vivante, assure la garde et la gestion d'un ensemble de plus de 100000 pièces, provenant des achats et commandes de l'État depuis la Révolution française. Le récolement des œuvres déposées par ses soins à l'École éclaire d'une manière originale les relations étroites que ces deux établissements, aux rôles éminemment complémentaires, ont entretenues aux xIX ${ }^{e}$ et $\mathrm{XX}^{\mathrm{e}}$ siècles ${ }^{1}$.

2 Fruit d'une politique régulière menée entre 1809 et 1923, les acquisitions et dépôts de l'ancienne administration des Beaux-Arts forment un corpus de plus de 15000 objets. À l'instar des envois annuels effectués par les pensionnaires de l'Académie de France à Rome, la plus grande partie d'entre eux a été attribuée pour servir de modèles d'instruction aux élèves et professeurs. Achetés et déposés le plus souvent sur 
proposition des directeurs de l'École, ces derniers étaient destinés à enrichir le « musée des études ", ouvert officiellement le 17 septembre 1834 au sein du palais éponyme, ainsi que les réserves de la bibliothèque.

Bien qu'elles s'inscrivent dans un remarquable esprit de continuité que les différentes réformes institutionnelles n'ont cessé de renforcer, les actions conduites par les bureaux chargés du soutien à la création contemporaine ${ }^{2}$ n'ont pas manqué de connaître de profondes évolutions. Celles-ci furent déterminées par deux facteurs principaux: la nature des demandes formulées par les directeurs et l'architecture des locaux dont la configuration a subi d'importantes modifications au cours du XIX ${ }^{e}$ siècle $^{3}$.

\section{L'âge d'or du néoclassicisme (1809-1863)}

4 Les premiers achats effectués par le ministère de l'Intérieur au profit de l'École remontent à la période impériale. Du Premier Empire à 1863, année d'une grande réforme, la majeure partie d'entre eux se compose de maquettes et de moulages auxquels s'adjoint un premier lot de copies de tableaux. La préférence accordée à ces artefacts, exécutés d'après des œuvres de l'Antiquité et de la Renaissance, illustre l'influence déterminante de la doctrine académique au sein d'une institution où la pédagogie repose alors essentiellement sur l'apprentissage du dessin enseigné d'après l'antique et le modèle vivant.

5 Comme le confirme une lettre du 4 avril 1809 adressée par le ministre de l'Intérieur Emmanuel Crétet de Champmol à Léon Dufourny, conservateur des collections, le modèle en liège du Colisée, exécuté entre 1792 et 1805 par l'architecte et archéologue italien Carlo Lucangeli (1747-1812), est la première œuvre attribuée à l'institution par les services de l'État ${ }^{4}$. Son installation, le 12 avril 1809, dans la galerie d'architecture, alors située au 10 rue de l'Université, inaugure une longue série d'achats qui illustre l'attention particulière accordée à cette catégorie d'objets par les collèges de professeurs.

6 En effet, excepté cette prestigieuse réalisation, 115 maquettes, réalisées en liège, en talc ou en plâtre, sont encore achetées et déposées par l'administration entre 1813 et 1839. Acquises auprès des collectionneurs Louis-François Cassas (1756-1827) ${ }^{5}$, Charles Texier $(1802-1871)^{6}$ et Auguste Pelet $(1785-1865)^{7}$, celles-ci doivent permettre aux peintres et architectes d'appréhender de façon claire et intelligible les beautés de l'architecture classique, comme en témoigne la réponse fournie par l'assemblée des enseignants le 8 avril 1808 au sujet de la collection Cassas :

Les dessins les mieux faits, les gravures les plus soignées ne peuvent à cet égard remplacer que d'une manière imparfaite, l'avantage inappréciable des modèles qui, faisant saillir à la fois toutes les formes les développent sur toutes les faces et les gravent dans la mémoire en traits ineffaçables sans obliger à cet effort d'attention générale qu'exige la comparaison de plans, de coupes, et d'élévations générales, difficiles à concevoir même pour l'artiste consommé... En même temps qu'elle facilitera l'étude des élèves, cette collection fournira au professeur des moyens précieux d'enseignement, il y puisera des exemples à proposer, des applications propres à éclairer les principes posés dans ses leçons, des démonstrations lumineuses qui achèveront de porter dans leur esprit le flambeau de l'évidence. Les élèves profiteront autant par cette méthode agréable, qui parle à l'esprit par les 
yeux, que par les commentaires, qu'il ne faut ni mépriser ni négliger, mais qui seuls

n'aident pas assez leur faible intelligence ${ }^{8}$.

7 Sous la monarchie de Juillet, peu après l'installation de l'École dans les locaux de l'ancien couvent des Petits-Augustins, une partie de ces chefs-d'œuvre en réduction est exposée dans la galerie de l'actuelle bibliothèque, située au premier étage du palais des Études. Présentés sur des épis en bois, ceux-ci offrent alors aux visiteurs un riche panorama de l'architecture antique où voisinent de nombreuses icônes de l'art grécoromain telles que le Parthénon, le temple de Poséidon à Paestum, le mausolée d'Halicarnasse, le portique du temple de Saturne à Rome (fig. 1) ou encore le théâtre d'Orange.

Figure 1

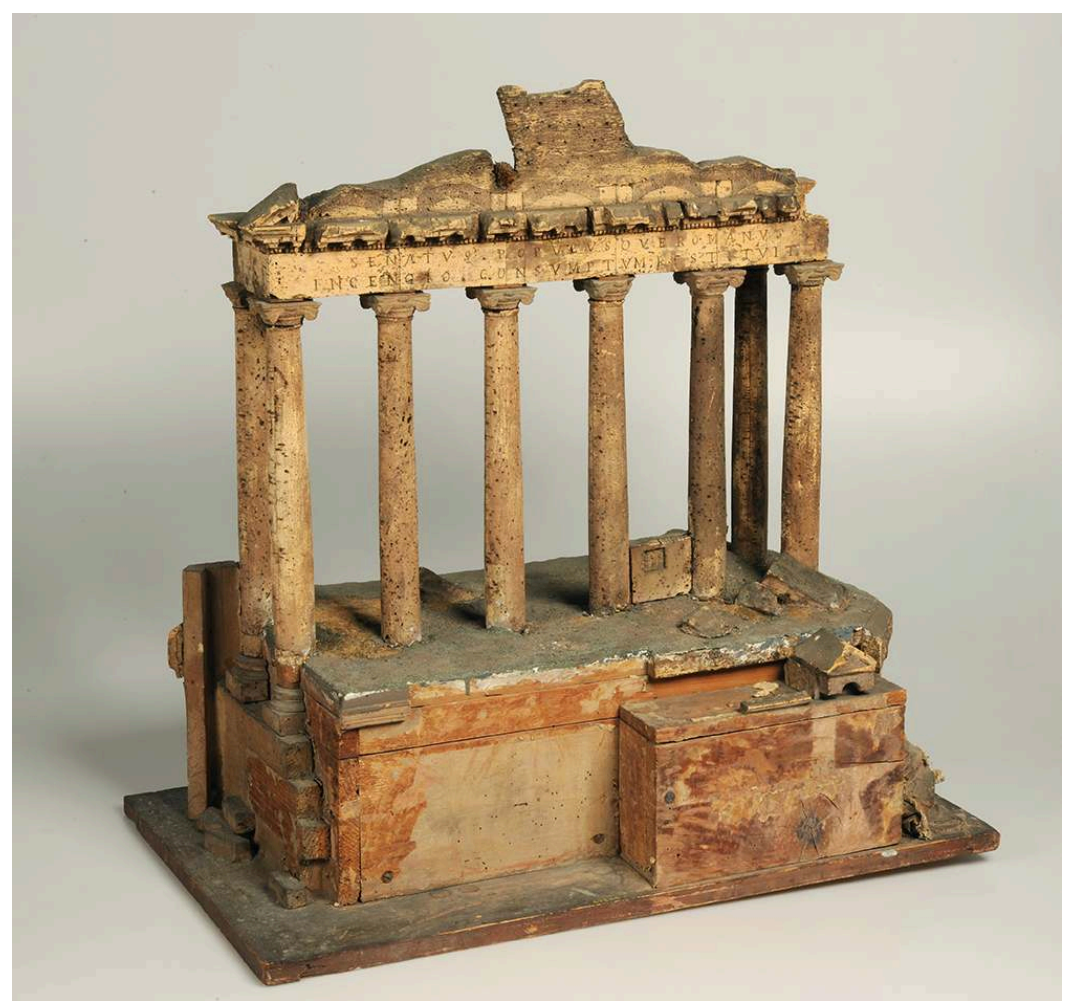

Giovanni Altieri. Portique du temple de Saturne à Rome. Seconde moitié du xvile siècle. Maquette d'architecture en liège. $\mathrm{H}: 0,71 ; \mathrm{L}: 0,70$. ENSBA, salle des copies. FNAC PFH-9022 (63).

(c) ENSBA.

Dès la fin des années 1830, la prédilection accordée à ces modèles, mis à la mode au XVIII ${ }^{e}$ siècle, décline néanmoins. Sous l'influence de Louis-Hippolyte Peisse (1803-1880), conservateur des collections depuis le 31 janvier 1835, et de Jean-Dominique Ingres (1780-1867), les achats effectués par l'État se concentrent progressivement sur les moulages. Ces derniers, considérés comme des supports d'enseignement privilégiés, tant pour les architectes que pour les sculpteurs, peintres et graveurs, sont alors destinés à appuyer les séances quotidiennes de dessin ou de modelage dites « d'après la bosse " prévues par le règlement constitutif de 1839, ainsi que les cours spéciaux d'anatomie, de perspective et d'histoire antique dispensés par les professeurs.

Expédiés en caisse depuis le port de Civitavecchia, sous le contrôle de l'Académie de France à Rome, ceux-ci sont le résultat de trois grandes campagnes d'acquisition ${ }^{9}$ qui 
permettent au musée de bénéficier de prestigieuses copies d'après l'antique (la Vénus du Capitole d'après Praxitèle, le Discobole de Myron, la Minerve de la villa Albani) (fig. 2) ainsi que de plusieurs modèles réalisés d'après les chefs-d'œuvre de la Renaissance italienne (Portes du baptistère de Florence d'après Andrea Pisano et Lorenzo Ghiberti, Frises de l'hôpital du Ceppo de Pistoia d'après Giovanni della Robbia, Tombeaux de Julien et Laurent de Médicis d'après Michel-Ange).

Figure 2

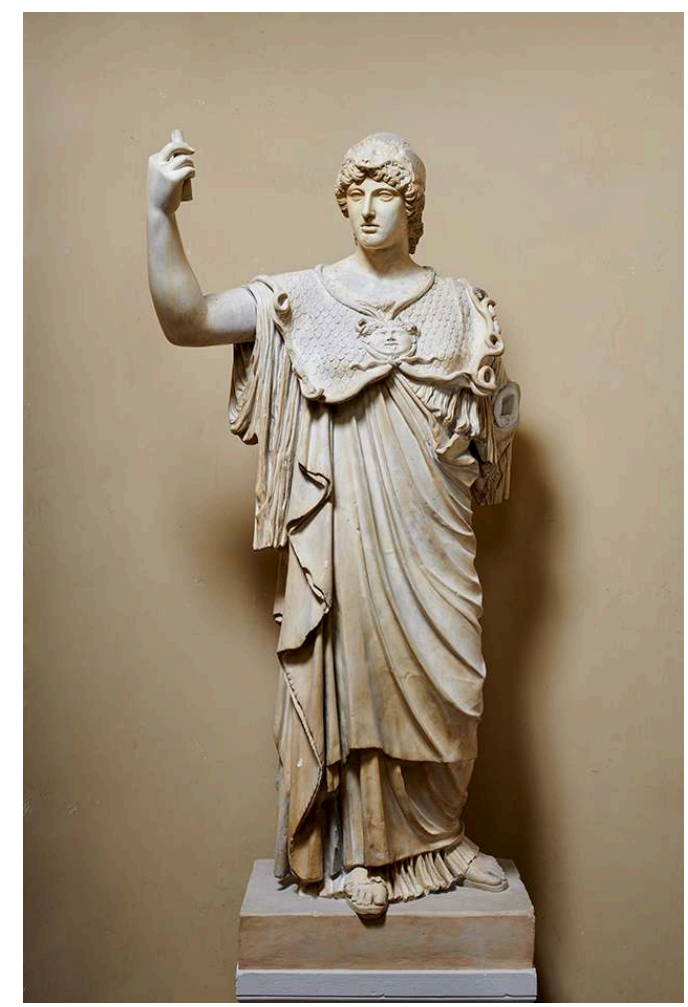

Minerve de la villa Albani (Rome). 1839. Ronde-bosse en plâtre. ENSBA, vestibule de la salle Melpomène. FNAC PFH-8918.

(c) ENSBA.

À partir de 1841, lors du retour de Jean-Dominique Ingres à la direction de l'École, la majeure partie des plâtres provient des investigations scientifiques menées en territoire hellénique. Entre 1843 et 1845, une quatrième expédition, confiée par le ministre de l'Intérieur Tanneguy Duchâtel à l'helléniste Philippe Lebas (1794-1860), accorde à l'établissement 187 moulages d'après le Parthénon, l'Érechtéion, le temple de Minerve Poliade et le monument chorégique de Lysicrate. Une dernière expédition, conduite entre 1860 et 1864 par l'archéologue François Lenormant (1837-1883), aboutit à l'attribution de nombreux exemplaires moulés d'après les découvertes effectuées sur le site d'Éleusis, dont le célèbre bas-relief Déméter et Perséphone remettant à Triptolème la royauté d'Éleusis (fig. 3). 
Figure 3

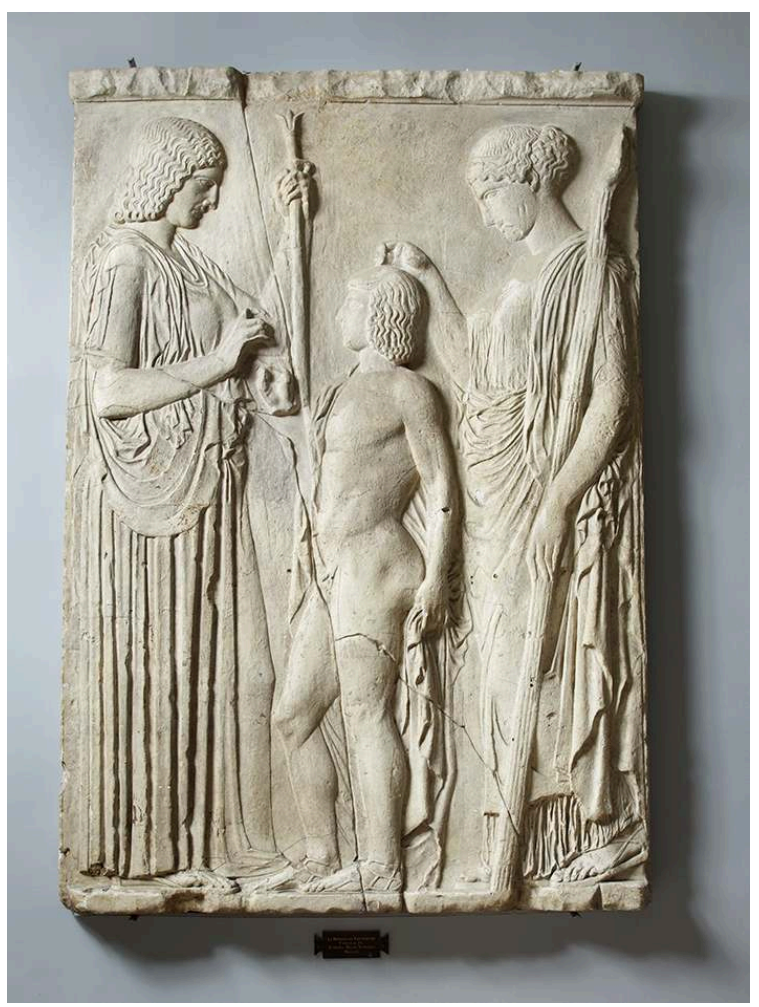

Déméter et Perséphone accordant à Triptolème la royauté d'Éleusis. Bas-relief en plâtre. Vers 1860. ENSBA, vestibule menant à l'hôtel de Chimay. FNAC PFH-8919.

(C) ENSBA.

11 L'ensemble de ces spécimens est alors déposé dans les salles ouvertes au rez-dechaussée du palais des Études (salle romaine, salle grecque, salle de l'ornement et salle d'Olympie) ainsi que dans la chapelle des Petits-Augustins, dont les locaux servent d'écrin aux œuvres dites modernes. Entre 1874 et 1876, en raison de l'engorgement rapide des salles du musée, une partie des œuvres est encore déployée dans la cour vitrée du palais, transformée par l'architecte Ernest Coquart (1831-1902) en lieu d'exposition des moulages antiques.

Par allèlement à ces attributions qui consacrent la prééminence des ouvrages en trois dimensions, une première série de toiles peintes est également attribuée par le ministère dès le règne de Louis-Philippe. Acheminées depuis Rome en format roulé, ces dernières offrent aux élèves la possibilité de contempler, pour la première fois, des copies de chefs-d'œuvre grandeur nature tels que le Jugement dernier de Michel-Ange (fig. 4), les Loges et les Chambres de Raphaël ou encore L'Assomption de la Vierge du Titien. 
Figure 4

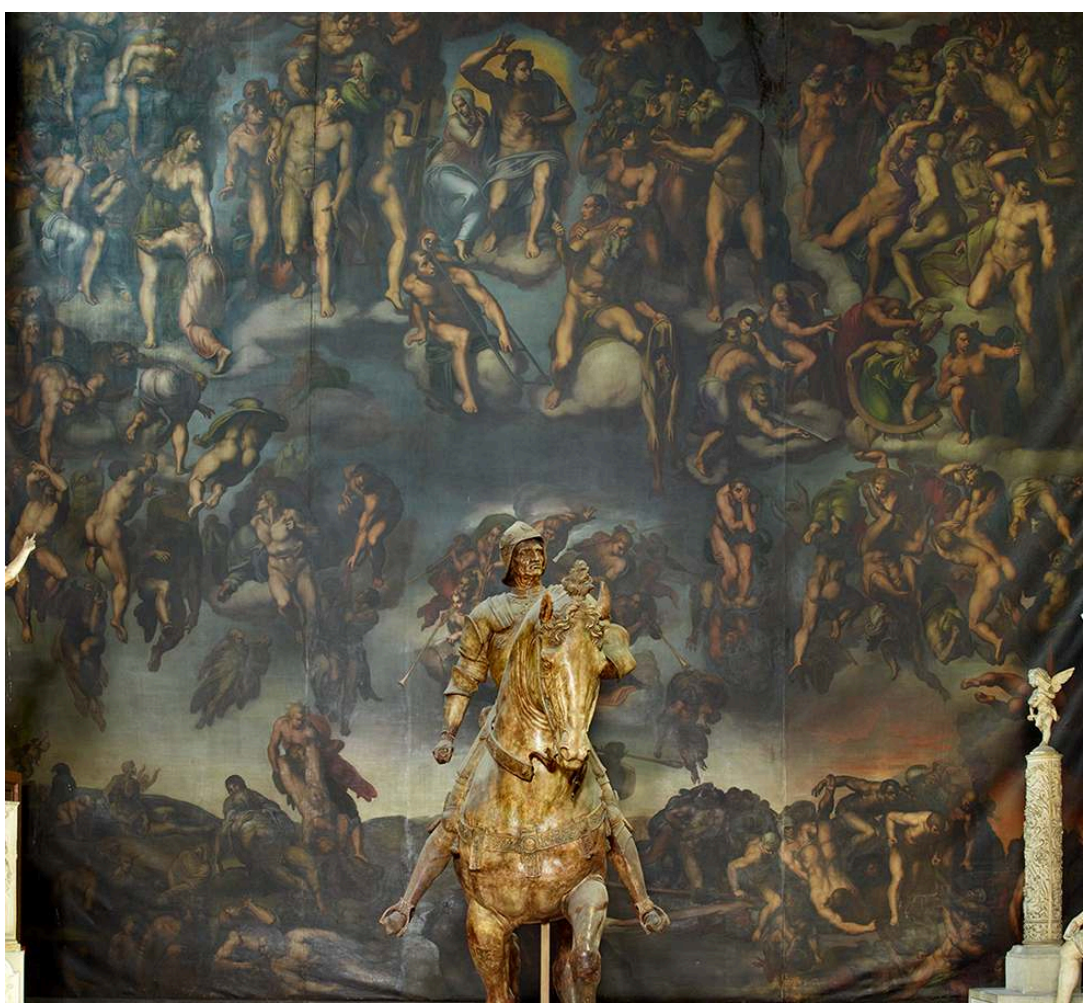

Xavier Alexandre François Sigalon. Le Jugement dernier, d'après Michel-Ange. 1837. Huile sur toile. $H: 690 ; L: 360$. ENSBA, chapelle des Petits-Augustins.

(c) ENSBA.

Saluées unanimement par les contemporains pour la qualité de leur exécution, bon nombre ont néanmoins des formats monumentaux qui ne sont pas sans poser des problèmes délicats d'exposition. En effet, si la version du Jugement dernier par XavierAlexandre Sigalon ${ }^{10}$ trouve place dans le fond de la chapelle des Petits-Augustins dès 1837, il n'en est pas de même des huit toiles exécutées d'après les Chambres de Raphaël par les frères Balze ${ }^{11}$, dont les mesures oscillent autour de 6 mètres de haut sur 9 mètres de large, ou encore de L'Assomption, qui atteint près de 7 mètres de haut. Les premières, suspendues aux murs du Panthéon à partir d'octobre 1847, réintègrent l'École, après leur décrochage en 1848 , en format roulé, et ne sont présentées aux yeux du visiteur qu'entre 1872 et 1873 , lors de leur transfert provisoire au musée des copies $^{12}$. Quant à L'Assomption d'après Titien, commandée au peintre d'histoire Henry Auguste Calixte Serrur (1794-1865) le 18 juillet $1844^{13}$ elle ne trouve un lieu de présentation définitif que lors de la construction de l'aile sur le quai Malaquais par Félix Duban (1797-1870), en 1862.

14 Ainsi, trente ans après la création du musée des études (1834), le bureau des Beaux-Arts a constitué une vaste collection de modèles dont les richesses doivent aider les élèves dans la quête du beau idéal, chère à Jean-Dominique Ingres. Les évolutions institutionnelles qui affectent l'École sous le Second Empire vont néanmoins modifier de manière substantielle les dispositions de ce schéma hérité des anciennes Académies. 


\section{Eugène Guillaume ou le temps de l'éclectisme (1864-1878)}

15 Avec l'adoption, le 13 novembre 1863, d'un nouveau décret relatif à l'organisation administrative et pédagogique de l'École ${ }^{14}$, la politique des dépôts menée par la direction des Beaux-Arts, désormais rattachée au ministère de la Maison de l'empereur, connaît une importante évolution.

Critiquant l'organisation traditionnelle du cursus scolaire fondé sur la hiérarchie des genres et le culte de l'antique, la nouvelle législation élaborée par le surintendant Alfred-Émilien de Nieuwerkerke (1811-1892), invite le corps enseignant à élargir la palette des références stylistiques proposées aux élèves, tout en exhortant les professeurs à accorder davantage de place à l'étude d'après nature. Dès 1864, ces recommandations se traduisent par une réforme de la scolarité, divisée en deux pôles : une partie pratique, ayant pour objectif le développement des qualités personnelles et individuelles de l'élève, et une partie théorique, vouée au perfectionnement de la culture historique et archéologique. La première donne naissance à la création d'ateliers, permettant aux étudiants d'aborder directement l'apprentissage de la peinture au sein de l'École ${ }^{15}$, tandis que la seconde contribue à l'institution de deux chaires, l'une d'esthétique et d'histoire de l'art, accordée à l'architecte Eugène Violletle-Duc (1814-1879), l'autre d'histoire et d'archéologie, attribuée à l'helléniste Léon Heuzey ${ }^{16}(1831-1922)$.

Bien que l'ensemble de ces mesures n'ait pas entraîné dans les années qui suivirent de changements profonds dans la production artistique des élèves, ces nouvelles dispositions eurent de facto un certain nombre d'incidences sur les modèles que l'État octroyait. En effet, si les moulages sont encore numériquement majoritaires ${ }^{17}$, au détriment des maquettes dont la mode disparaît quasi définitivement ${ }^{18}$, d'autres formes matérielles sont désormais privilégiées. Sous l'impulsion du peintre romantique Joseph-Nicolas-Robert Fleury (1797-1890) puis du sculpteur Eugène Guillaume (1822-1905), directeur de 1866 à 1878, l'École accueille un lot considérable de tableaux, dessins et photographies qui, tout en diversifiant les supports d'enseignement, offrent aux élèves de nouvelles sources d'inspiration. À la différence de la période précédente, les champs historiques et géographiques s'élargissent considérablement, favorisant l'émergence d'une culture désormais plus diverse au sein de laquelle l'Antiquité grécoromaine et le $\mathrm{XvI}^{\mathrm{e}}$ siècle italien ne constituent plus les seuls champs de référence. D'autre part, la création contemporaine, inspirée par le romantisme et le naturalisme, fait l'objet de plusieurs dépôts remarquables, satisfaisant ainsi le désir des promoteurs de la réforme d'une ouverture à des sensibilités diverses.

143 toiles, issues majoritairement du musée européen des Copies créé par le directeur des Beaux-Arts Charles Blanc en 1871 (1813-1882) ${ }^{19}$, rejoignent les cimaises de la rue Bonaparte au cours de cette période. Leur accrochage, en 1875, dans les salles du palais des Études et dans la nouvelle aile du quai Malaquais, bouleverse la typologie des modèles traditionnellement exposés. En effet, si sur les 134 peintures transférées à la demande d'Eugène Guillaume, 91 sont encore des copies effectuées d'après des maîtres italiens ou des fresques antiques (fig. 5), 38 autres s'inspirent de maitres allemands, espagnols, flamands, français et hollandais. 
Figure 5

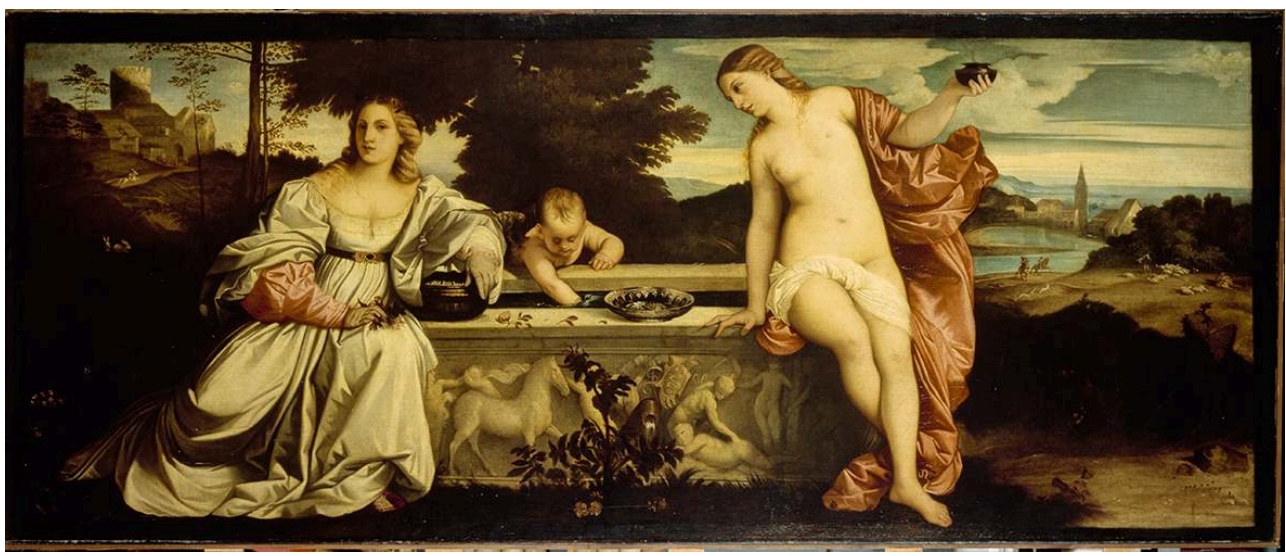

Hector Leroux. L'Amour sacré, l'Amour profane d'après Titien. 1860. Huile sur toile. H : 150 ; L : 150. ENSBA, secrétariat de l'hôtel de Chimay. FNAC FH 860-151.

(c) ENSBA.

19 Une collection inédite, dont certains exemplaires n'étaient encore connus que par la gravure, s'offre ainsi aux regards des disciples où le classicisme italien voisine avec le ténébrisme de Ribera (fig. 6), le colorisme vigoureux de Rubens, la poésie lumineuse des scènes de Rembrandt, le réalisme truculent et la touche franche de Vélasquez (fig. 7).

Figure 6

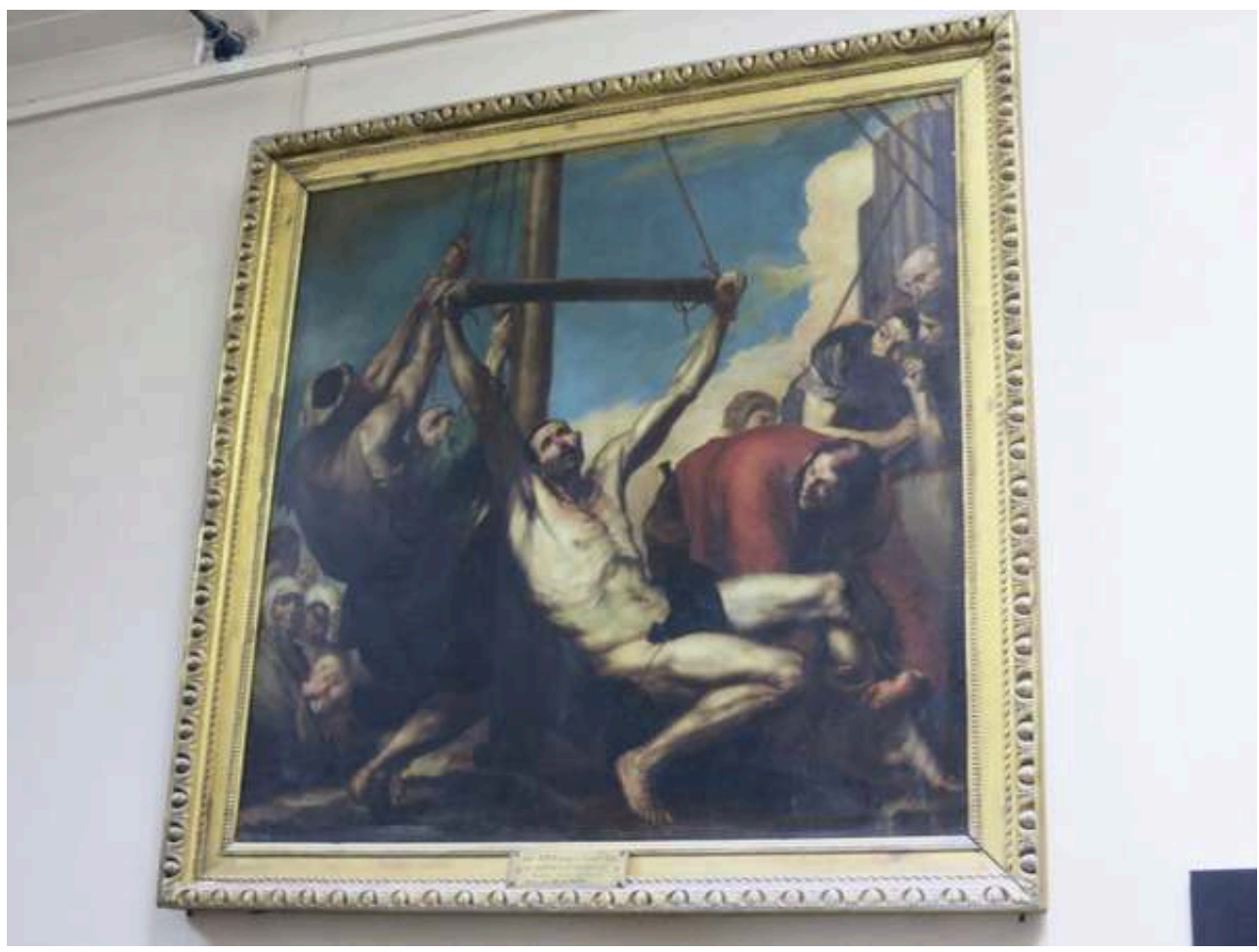

Joseph Fortuné Layraud. Le Martyre de saint Barthélémy, d'après Ribera. 1873. Huile sur toile. H : 239 ; L : 235. ENSBA, amphithéâtre d'anatomie. FNAC PFH-836.

(c) ENSBA. 
Figure 7

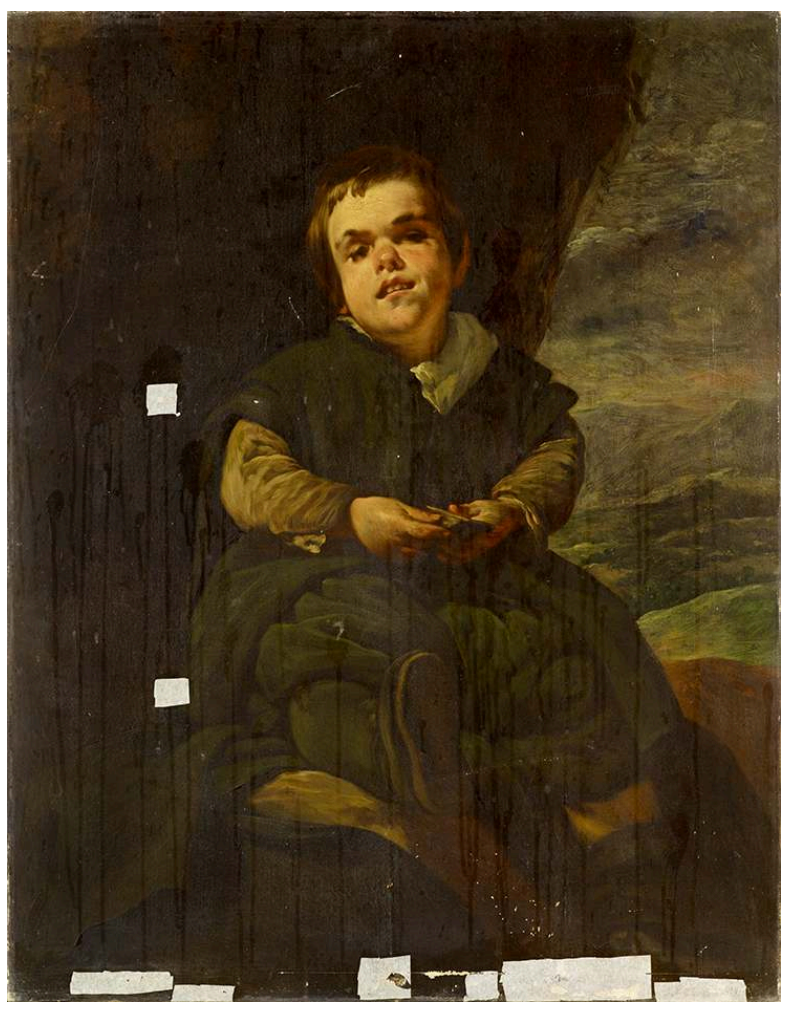

Jean-Baptiste Guignet. Le Nain de Vallegas, d'après Diego Vélasquez. Vers 1850. Huile sur toile. H: 110,$5 ; \mathrm{L}: 85,5$. ENSBA, salle des copies du palais des Etudes. FNAC PFH-8320.

(c) ENSBA.

Défiant la traditionnelle hiérarchie des genres, plusieurs portraits et scènes pittoresques sont montrés, aux côtés des sujets inspirés par l'Histoire et la fable. En 1889, selon Eugène Müntz, auteur d'un Guide de l'École nationale des beaux-arts, 11 tableaux d'après Frans Hals (Les Officiers du tir de Saint-Georges), Paulus Potter (Le Taureau), Rembrandt (Les Syndics de la corporation des drapiers) et Vélasquez (Les Quatre Bouffons, Les Fileuses, La Dame au gant, La Famille de Philippe IV et Le Portrait équestre de Philippe IV) ornent ainsi les murs de la salle de la charpente ${ }^{20}$ tandis que deux autres, L'Officier de fortune de Rembrandt et le Repas des gardes civiques de Bartholomeus Van der Helst côtoient en salle Schoelcher Le Mariage mystique de sainte Catherine du Corrège et $L a$ Venise triomphante de Véronèse.

21 Hormis ce dépôt exceptionnel, 1756 dessins sont également concédés à la bibliothèque ouverte au public depuis le 24 janvier 1864 sous la direction d'Ernest Vinet ${ }^{21}$ (1804-1878). Leur attribution répond à un triple objectif: valoriser la création contemporaine, améliorer la culture historique et archéologique des futurs artistes et fournir aux maîtres dirigeant les ateliers pratiques de nouveaux modèles d'apprentissage susceptibles d'enrichir les savoir-faire.

Une part appréciable du corpus est alors constituée par des travaux d'élèves, réalisés dans le cadre du concours pour le Prix de Rome ou lors du séjour à la villa Médicis. Il en est ainsi des projets architecturaux qui ont remporté les Seconds Grands Prix ${ }^{22}$ (fig. 8), que les services de l'État acquièrent régulièrement à partir de 1865, ainsi que des recueils d'études dus à des «Premiers Grands Prix » lors de leur séjour ultramontain ${ }^{23}$. 
Figure 8

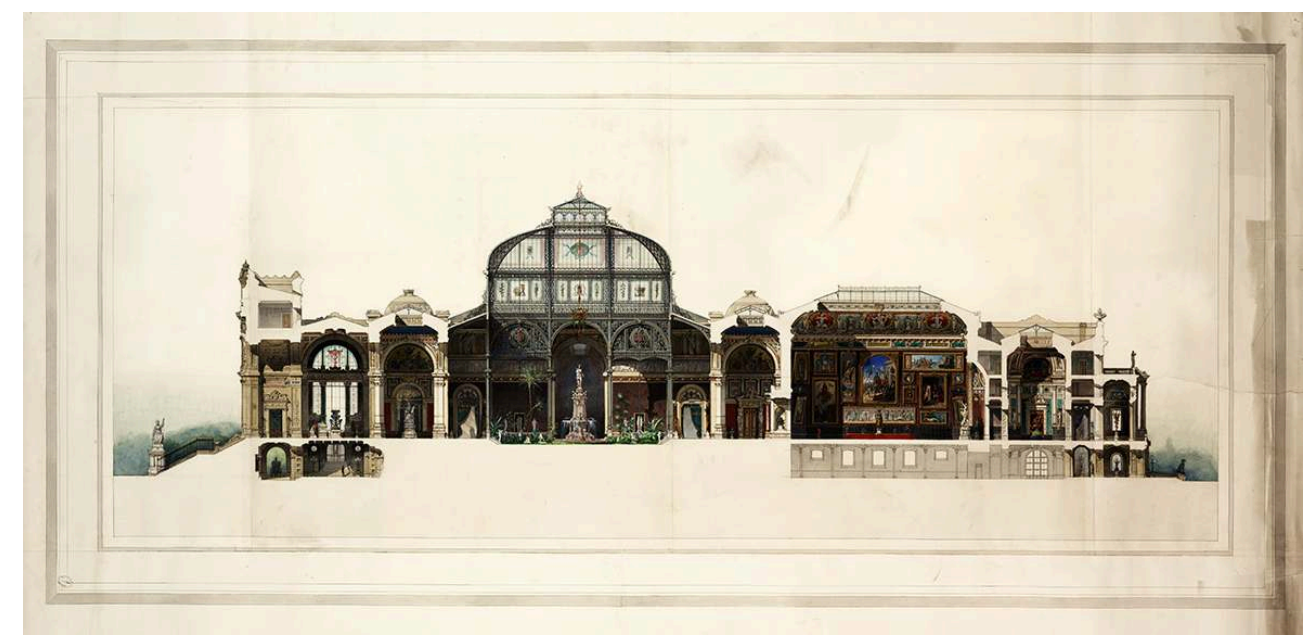

Pierre-Henri Mayeux. Un Palais pour l'exposition des Beaux-Arts. 1er Second Grand Prix de Rome, 1867. Crayon, encre de Chine et aquarelle sur papier. $H: 60,3 ; L: 99,5$. ENSBA, salle du premier étage du palais des Études. FNAC FH 867-212 (5).

(c) ENSBA.

Vingt-six Seconds Grand Prix sont achetés entre 1865 et 1878, au prix de 1500 francs

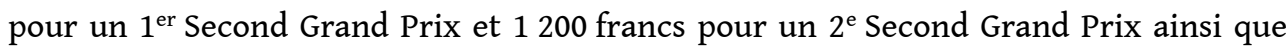
trois albums d'études majeurs: ceux de Jacques-Martin Tétaz (1818-1865) sur la basilique de Palestrina ${ }^{24}$, d'Alexis Paccard (1813-1867) sur le Parthénon ${ }^{25}$ et de JeanJacques Clerget (1808-1877) sur les monuments de la Grèce, d'Italie et d'Asie mineure ${ }^{26}$ (fig. 9). Acquis auprès des héritiers des artistes, ces trois recueils fournissent un répertoire précieux de formes et de modèles pour les cours d'esthétique dont l'un, assuré entre 1864 à 1884 par Léon Heuzey, est entièrement consacré à l'art grec et aux mythes anciens. Composés de nombreuses esquisses et dessins préparatoires consacrés parfois à des monuments encore peu connus, ils mettent ainsi à l'honneur le travail préliminaire des artistes, aux côtés d'œuvres plus achevées telles que les envois de $4^{\mathrm{e}}$ année. 
Figure 9

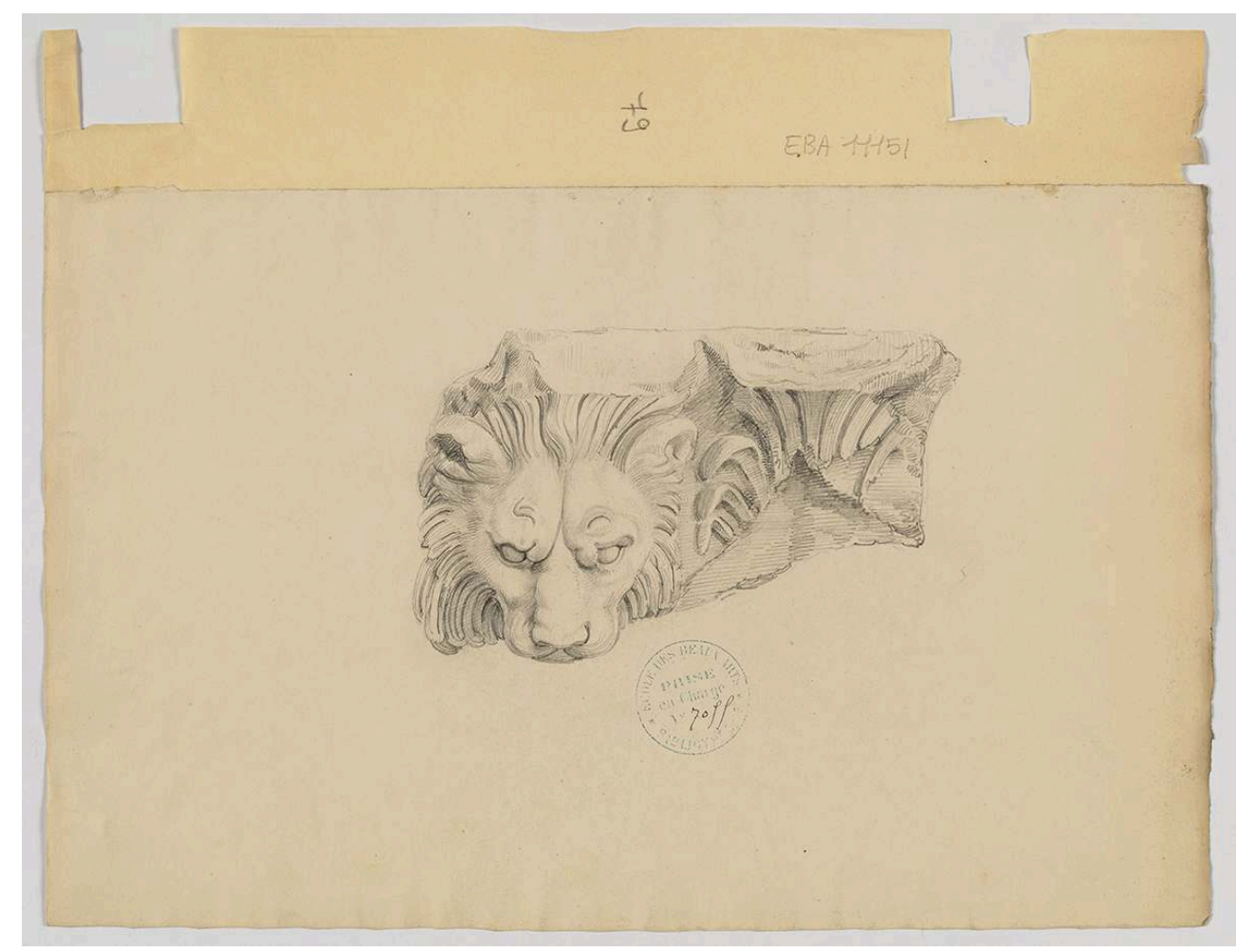

Jean-Baptiste Clerget. Fragment de sculpture avec tête de lion. Vers 1840. Crayon sur papier. H : 21,3; $\mathrm{L}:$ 33,3. ENSBA, bibliothèque du palais des Études. FNAC PFH-8310 (454).

(c) ENSBA.

24 À l'opposé de ces réalisations, dont les références relèvent encore de la tradition néoclassique, une autre partie du lot réunit des études préparatoires réalisées par des peintres et des sculpteurs d'obédience romantique. Certaines, issues de recherches personnelles ou d'observations effectuées lors de lointaines pérégrinations, promeuvent un dessin à caractère scientifique, inspiré par l'idée du rapprochement entre l'art et la science si chère à de nombreux contemporains. 
Figure 10

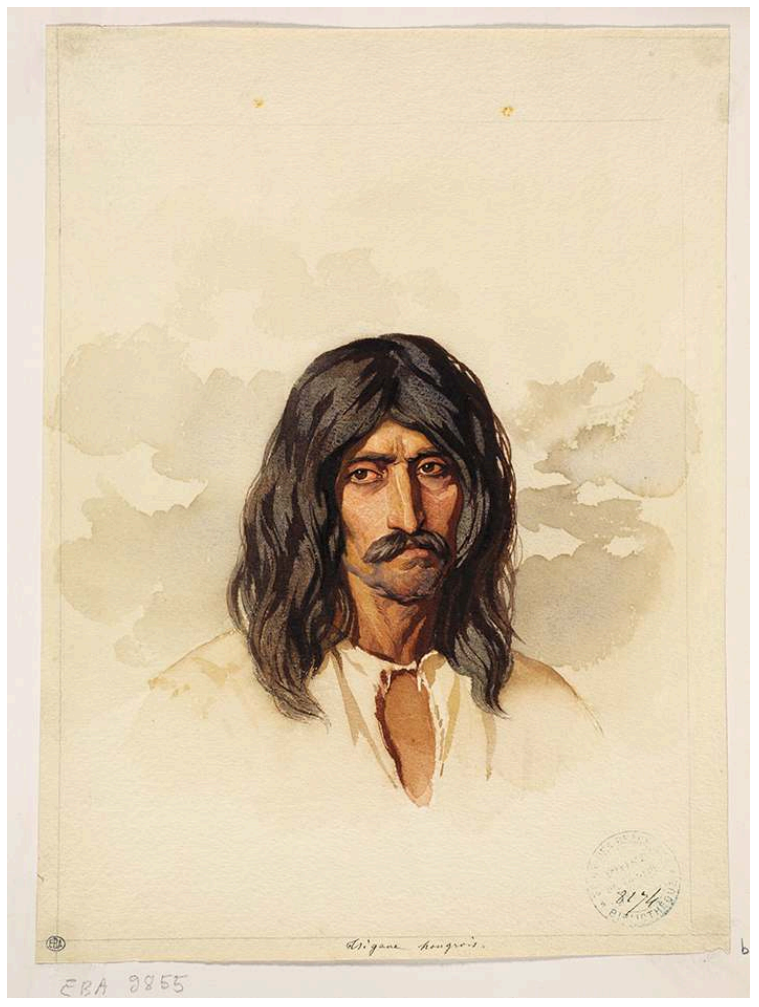

Théodore Valério. Tzigane des environs d'Arad. 1851. Crayon et aquarelle sur papier. H: 26,8; L : 20,6. ENSBA, bibliothèque du palais des Études. FNAC PFH-8798 (57).

(c) ENSBA. 


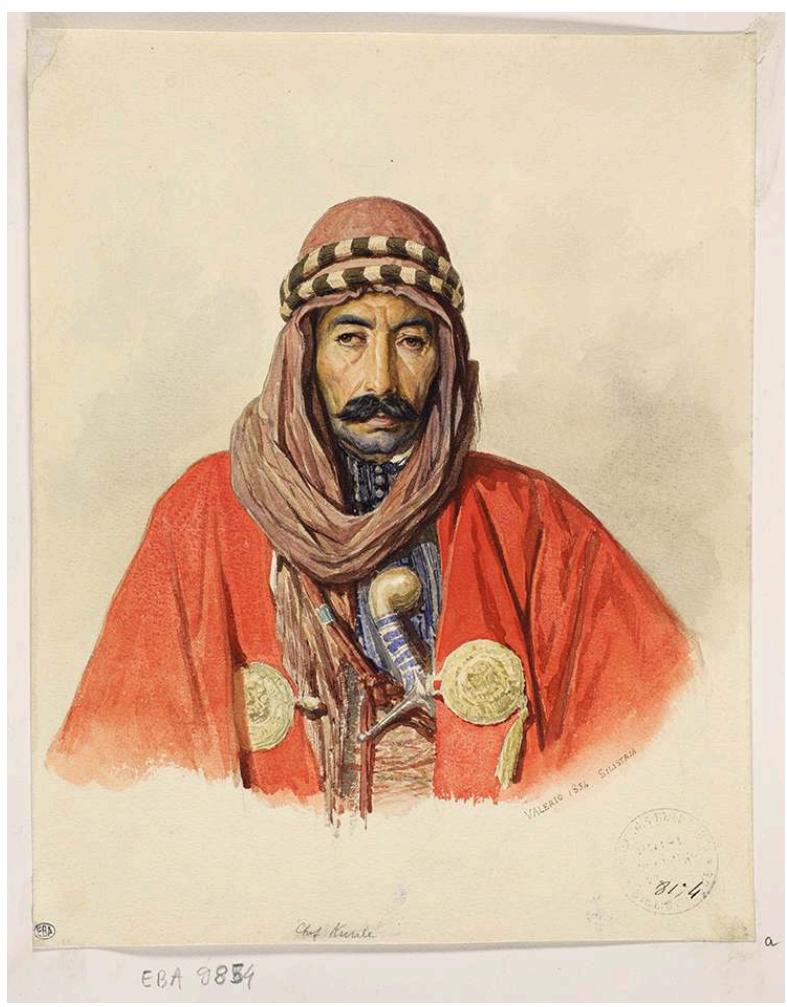

Théodore Valério. Homme de Silistrie. 1854. Crayon et aquarelle sur papier. $H: 28,1 ; L: 22,3$. ENSBA, bibliothèque du palais des Études. FNAC PFH-8798 (83).

\section{(c) ENSBA.}

C'est ainsi que le 30 mars 1869, l'attribution de 100 esquisses au crayon et 90 aquarelles de l'artiste-voyageur Théodore Valério (1819-1879) ${ }^{27}$, introduit les artistes au dessin ethnographique d'inspiration orientaliste (fig. 10, fig. 11). Apparu autour des années 1830, lorsque s'impose la mode des voyages pittoresques en Orient, le mouvement connaît un regain d'intérêt sous le Second Empire. Plusieurs événements et manifestations en témoignent alors, telles que la présentation au sein de l'Exposition des produits de l'Algérie en 1860 de la «Galerie ethnographique et anthropologique pour servir à l'histoire des races ${ }^{28}$, série de bustes, souvent polychromes, de Charles Cordier (1827-1905), ou la nomination, en 1863, du peintre Jean-Léon Gérôme (1824-1904) à la tête de l'un des ateliers pratiques de l'École. Fondateur du courant néogrec et partisan d'une antiquité renouvelée débarrassée des modes de représentation académiques, ce dernier ne tarde pas à développer une peinture à prétention scientifique, orientée vers une production de style anthropologique qui lui vaudra aussitôt le surnom de peintre ethnographe.

Influencés par les efforts de catalogage des races et des types qui composent l'humanité entrepris dans certains milieux scientifiques, les dessins de Valério forment une exceptionnelle galerie de portraits exécutés au cours de trois voyages accomplis aux confins de l'Europe orientale entre 1851 et 1854 : les deux premiers en Hongrie et le troisième dans les provinces danubiennes, où l'artiste observa les populations mahométanes de l'Asie et de l'Afrique rassemblées par l'armée irrégulière turque pendant la guerre de Crimée. Fruit d'une attention toute particulière aux types physiques et aux tenues vestimentaires, ils constituent une illustration vivante et 
colorée des différents peuples qui composent l'empire d'Autriche et l'Empire ottoman à la veille des grands bouleversements suscités par la révolution industrielle. Hongrois de la plaine, Tziganes des Carpates, Valaques de Transylvanie ou encore Slaves des frontières militaires et de Bosnie y sont minutieusement dépeints sous les traits de personnages altiers dont la mélancolie n'est pas sans rappeler l'adhésion précoce de l'artiste au courant romantique.

Quelques années plus tard, le dépôt des œuvres conservées dans l'atelier d'Antoine Barye (1795-1875), fournit à l'École un autre corpus qui témoigne, de manière tout aussi remarquable, de l'influence exercée par la science sur la création artistique ${ }^{29}$. Acquis par l'administration des Beaux-Arts lors de la vente des œuvres du sculpteur à l'hôtel Drouot en février 1876, le fonds réunit alors 170 croquis à la plume et 70 moulages effectués d'après des animaux morts, à la suite de nombreuses séances au Museum d'histoire naturelle. Lions, tigres, cerfs, bouquetins ou tortues, protagonistes habituels du bestiaire de l'artiste, y sont représentés sous la forme de simples silhouettes dont les contours sont accompagnés de notations et de mesures mathématiques donnant à l'artiste une idée parfaite de la forme et des proportions (fig. 12).

Figure 12

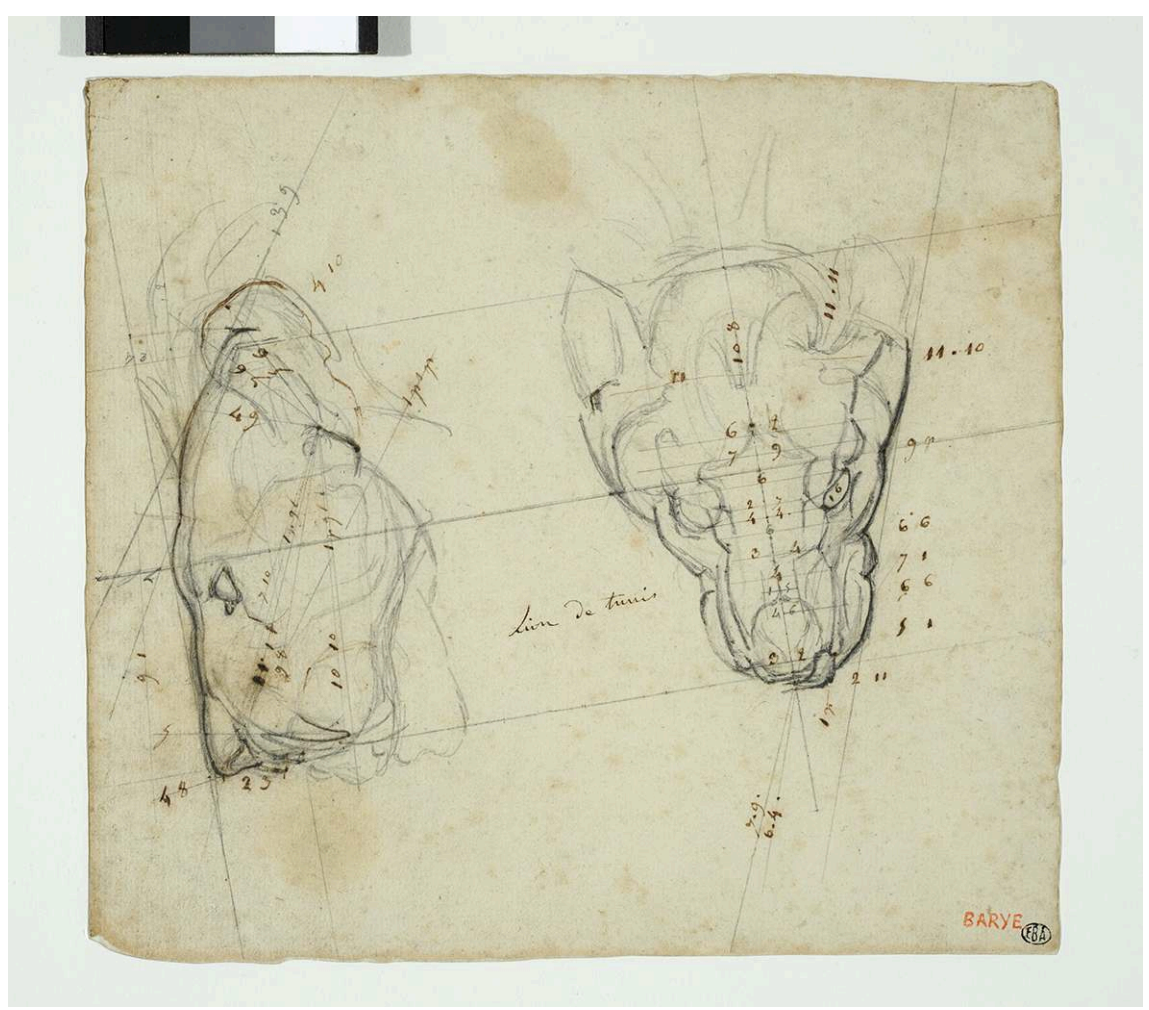

Antoine Barye. Lion de Tunis. Mine de plomb et encre brune sur papier. $H: 17,6$; L : 19,4. ENSBA, bibliothèque du palais des Études. FNAC PFH-8533 (17).

(c) ENSBA.

L'ensemble, inspiré par les études de l'historien de l'art Toussaint-Bernard ÉmericDavid (1755-1839) sur l'art statuaire chez les anciens et les modernes ${ }^{30}$, ne tarde pas à devenir un objet d'étude majeur pour tous les artistes animaliers, satisfaisant ainsi les vœux exprimés par le professeur d'anatomie Mathias Duval (1844-1907) à l'origine de cette acquisition : 
En visitant, en étudiant, il y a quelques semaines, l'exposition si instructive des œuvres de Barye, j'ai été, comme tout le monde, vivement impressionné en voyant, dans la première salle de l'exposition, ces dessins avec échelles à proportions, ces moulages sur nature, ces mensurations qui nous montrent avec quelle patience et quelle conscience l'artiste avait analysé les divers sujets qu'il devait reproduire. Quel plus bel exemple donner à nos élèves que celui de ces études profondes de la part d'un artiste qui semble au premier abord avoir deviné la nature tant il la reproduite pleine de vie et de vérité. S'il est vrai, ainsi que je l'ai entendu dire, qu'une vente doit disperser ces précieuses études par le dessin et le moulage, il serait infiniment heureux que l'École des beaux-arts put faire l'acquisition des principales pièces et dessins pour la galerie anatomique. Notre collection, déjà fort sérieuse, s'élèverait ainsi définitivement à une importance digne de l'École des beaux-arts ${ }^{31}$.

Bien que la culture gréco-romaine et l'art italien dominent encore largement le spectre des acquisitions, de nouveaux courants artistiques parviennent donc à s'imposer au cours de cette période. La Troisième République, en dépit de son attachement aux valeurs traditionnelles prônées par les générations précédentes, ne manque pas de poursuivre dans cette voie, diversifiant encore le catalogue des modèles proposés aux artistes.

\section{Entre patrimoine national et monde extra-européen : les dernières politiques de dépôt au temps de Paul Dubois et Léon Bonnat (1879-1922)}

Sous la direction de Paul Dubois (1829-1905) et Léon Bonnat (1905-1922), respectivement directeurs entre 1878 et 1905 et de 1905 à 1922, la politique conduite par le ministère connaît encore une nouvelle inflexion. Placée depuis 1874 sous la tutelle du ministère de l'Instruction publique, la direction des Beaux-Arts cesse progressivement ses acquisitions dans le domaine des moulages et des maquettes, considérés comme des modèles d'inspiration obsolètes, tout en réduisant considérablement ses achats dans le domaine de la copie peinte ${ }^{32}$. En liaison avec le développement du culte des grands hommes et la création de nouvelles chaires d'enseignement, les acquisitions et les dépôts se concentrent essentiellement sur la statuaire à vocation commémorative ainsi que sur les arts graphiques.

Destiné à pallier l'absence de budget affecté à l'achat et à la commande d'œuvres d'art au sein de l'institution, le soutien accordé par l'État au développement de la sculpture remonte au Second Empire et aux débuts de la Troisième République. Dès cette époque, l'administration finance en effet l'exécution de plusieurs œuvres dédiées à des personnalités ayant marqué l'histoire des lieux tels l'archéologue et historien Alexandre Lenoir (1761-1839) ${ }^{33}$, le peintre orientaliste Henri Regnault (1843-1871) (fig. 13), le graveur-médailleur Jean-Baptiste-Eugène Farochon (1812-1871) ${ }^{34}$ (fig. 14) ou encore les architectes Alphonse-François Marie Jaÿ (1789-1871) $)^{35}$ et Auguste Rougevin (1831-1856) ${ }^{36}$. 
Figure 13

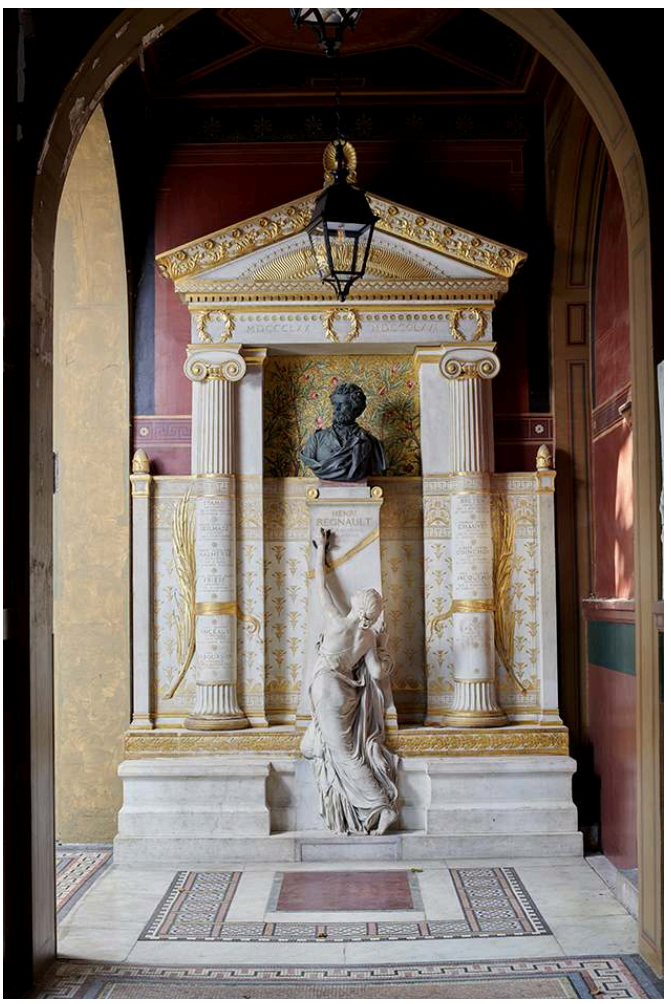

Charles Degeorge et Henri Chapu. Buste d'Henri Regnault et allégorie de la Jeunesse. 1876. Ronde-bosse en bronze et marbre. H: 72 ; L : 62 ; Pr : 44 - H : $201 ; \mathrm{L}: 62 ; \mathrm{Pr}: 40$. ENSBA, cour du Mûrier. FNAC PFH-8244 et FNAC PFH-8524.

(C) ENSBA 
Figure 14

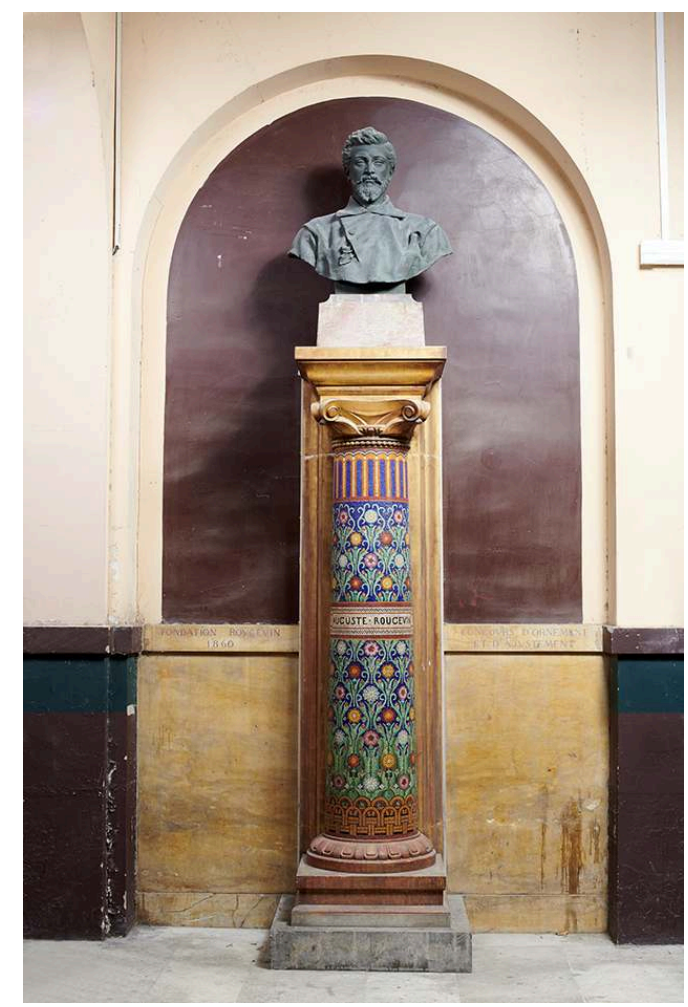

Ernest Guilbert. Auguste Rougevin. 1884. Bronze. Dimensions non prises. ENSBA, vestibule de la cour du Mûrier conduisant à l'aile du quai Malaquais. FNAC PFH-8525.

(c) ENSBA.

Les monuments qui leur sont consacrés adoptent la forme d'un simple buste, traité en pierre ou en bronze, à l'exception de celui dressé en 1876 en l'honneur d'Henri Regnault, dont la composition est plus ambitieuse. Issu d'une collaboration entre les architectes Jean-Louis Pascal (1837-1920) et Georges-Ernest Coquart ${ }^{37}$ (1831-1903), chargés de la partie architecturale, et les sculpteurs Charles Degeorge (1837-1888) et Henri Chapu (1833-1891) auteurs du buste et de l'allégorie de la Jeunesse ${ }^{38}$, l'ensemble met en scène, dans un cadre architectural en forme de temple très orné, une jeune fille éplorée tendant un rameau de laurier à l'artiste défunt, tué lors de la bataille de Buzenval le 19 janvier 1871.

À partir de 1891, la volonté de l'établissement de constituer au sein des salles de l'hôtel de Chimay, acquis en 1884, une vaste collection de portraits d'artistes relance cette première implication de l'administration dans le domaine de la sculpture. Créée à l'initiative du sculpteur Paul Dubois sur le modèle de la galerie des portraits des Offices à Florence, celle-ci a pour vocation d'accueillir l'image des plus éminents professeurs de l'Institution selon un ordre chronologique privilégiant trois périodes : une première

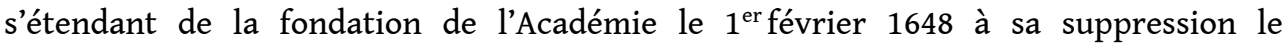
28 septembre 1793, une seconde allant de 1793 au 13 novembre 1863, date de l'arrêt modifiant le fonctionnement de l'établissement, la dernière débutant de 1863 jusqu'à l'époque contemporaine. 
Figure 15

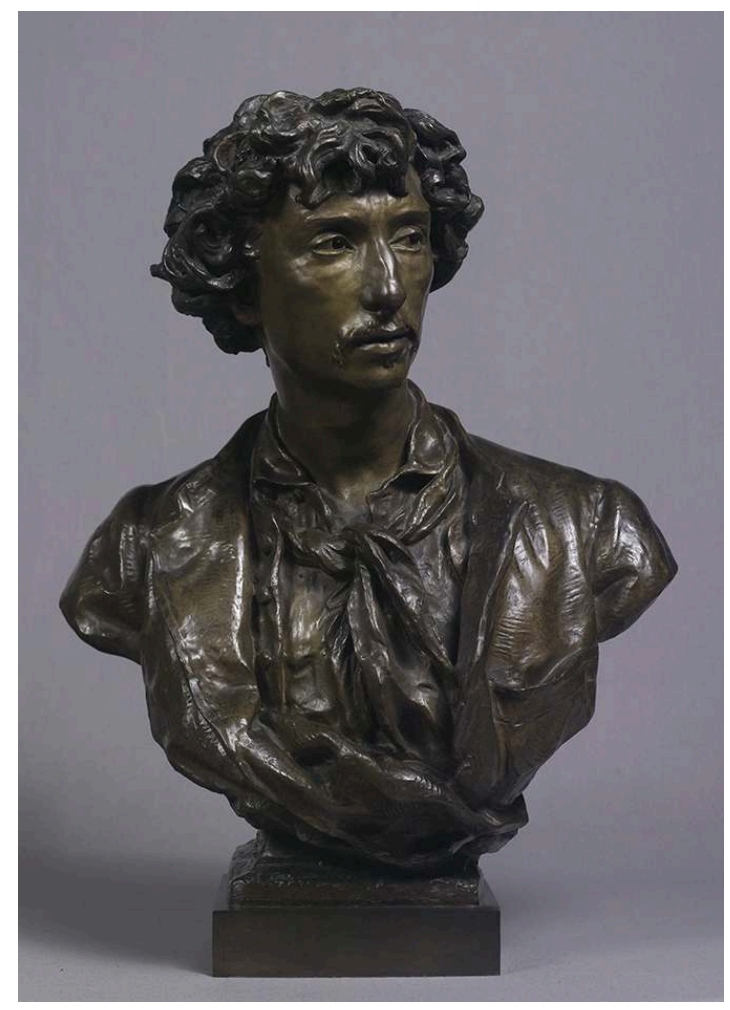

Edmond Gruet. Charles Garnier, d'après Jean-Baptiste Carpeaux. 1895. Bronze. H : 74 ; L : 57 ; Pr : 28. ENSBA, salle des copies du palais des Études. FNAC 1158.

\section{(C) ENSBA.}

Entre 1892 et 1914, 13 bustes $^{39}$ commandés par l'État rejoignent ainsi cette nouvelle galerie installée dans la salle du conseil et la salle Louis XIV. Placés sur des gaines aux côtés de nombreux portraits peints ou dessinés, tous rendent hommage à des personnalités du siècle célèbres par leur engagement dans l'enseignement et la vie de l'École, tels l'architecte Charles Garnier (1825-1898) ${ }^{40}$ (fig. 15), les peintres Paul Baudry (1828-1886), Léon Bonnat (1833-1922, Henri Delaborde (1811-1899), Guillaume GuillonLethière (1760-1832) (fig. 16) et Hippolyte Flandrin (1809-1864), les sculpteurs Claude Dejoux (1732-1816), Jean-Pierre Cortot (1787-1843), François Jouffroy (1806-1882) et Alexandre Falguière (1831-1900), le graveur Louis-Pierre HenriquelDupont (1797-1892), le professeur d'anatomie Mathias Duval (1844-1907) (fig. 17) et le mathématicien Pierre-Ossian Bonnet (1819-1892). 
Figure 16

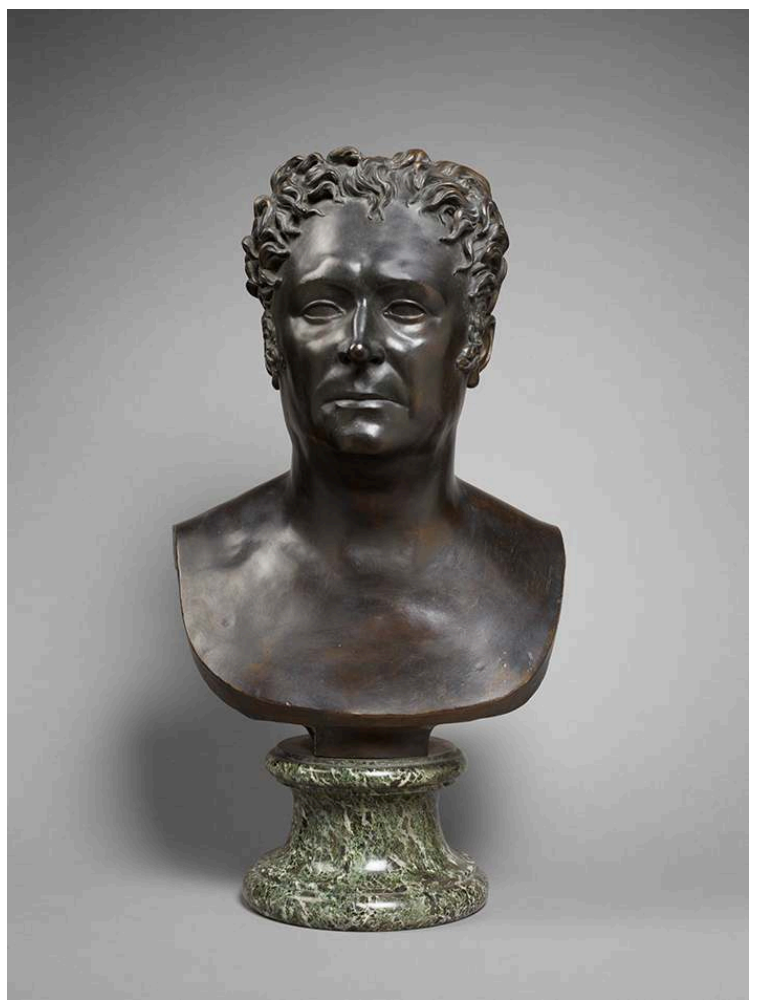

Edmond Gruet. Guillaume Guillon Lethière. 1895. Bronze. H : 63 ; L : 32. Pr : 28. ENSBA, salle Lesoufaché, palais des Études. FNAC PFH-8294.

(c) ENSBA. 
Figure 17

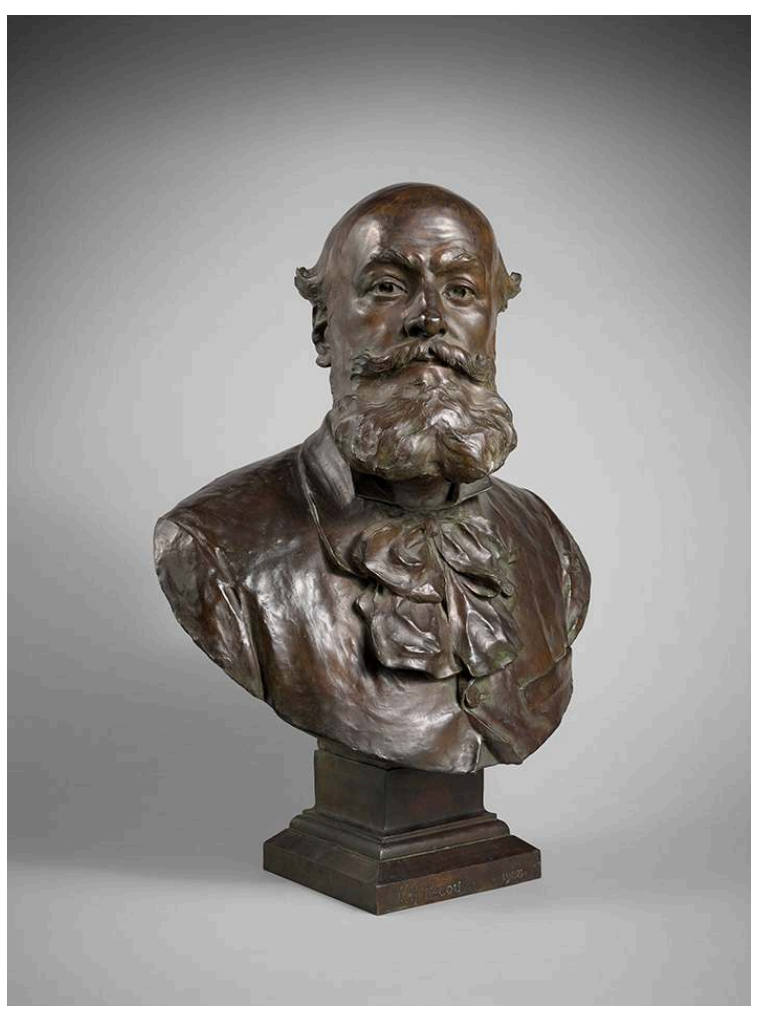

William Henri Pécou. Mathias Duval. 1910. Bronze. H : 55 ; L : 54 ; Pr : 26. ENSBA, salle Stratis Andréadis, palais des Études. FNAC 2374.

(c) ENSBA.

À côté de ces œuvres, dont la présence doit concourir à l'éducation morale et civique du personnel de l'établissement, 2831 dessins sont également octroyés à la bibliothèque, parmi lesquels 2005 dessins d'architectes et 826 dessins de maîtres. Alors que ces derniers, acquis auprès des héritiers des peintres Claude-Ferdinand Gaillard $(1834-1887)^{41}$ ou Jules-Élie Delaunay (1828-1891) ${ }^{42}$ témoignent d'un attachement vivace à la tradition classique, les dépôts effectués dans le domaine du dessin d'architecture révèlent un intérêt nouveau pour le vocabulaire des arts décoratifs de l'art du Moyen Âge et des Temps modernes.

Deux phénomènes conjoints paraissent avoir été directement à l'origine de cette évolution: l'intérêt porté à l'étude du patrimoine national, dont une partie des richesses est exposée aux yeux des visiteurs depuis l'ouverture du musée de Sculpture comparée en 1879 par Eugène Viollet-le-Duc, et la création au sein de l'École, le 14 mai 1873, de cours supérieurs d'arts décoratifs. Dispensés par le peintre Pierre-Victor Galland (1822-1892) puis par l'architecte Pierre-Henri Mayeux (1845-1929) à partir de 1892, ces derniers incitent en effet leurs élèves à dessiner d'après les chefs-d'œuvre des arts décoratifs de tous les temps et de toutes les civilisations. Bien qu'ils n'aient pas eu la fréquentation espérée en raison des préjugés de bon nombre de professeurs à l'égard de cet enseignement, leur tenue pousse les directeurs à favoriser le dépôt de recueils permettant d'appuyer les démonstrations effectuées lors des séances.

Deux albums majeurs font à ce titre leur entrée dans les collections entre 1893 et 1901, à l'initiative de Paul Dubois : les 836 relevés, dessins et aquarelles des architectes HenriLouis Lafillée (1859-1947) ${ }^{43}$ et Pierre Gélis-Didot (1853-1937) ${ }^{44}$, destinés à illustrer les 
ouvrages consacrés à l'histoire de la peinture monumentale en France du XI ${ }^{\mathrm{e}}$ au XVIII siècle, ainsi que les 1176 croquis d'architecture, de menuiseries, de mosaïques de la ville du Caire de l'architecte Jules Bourgoin (1838-1908), ancien professeur d'ornement à l'École des beaux-arts ${ }^{45}$.

Figure 18

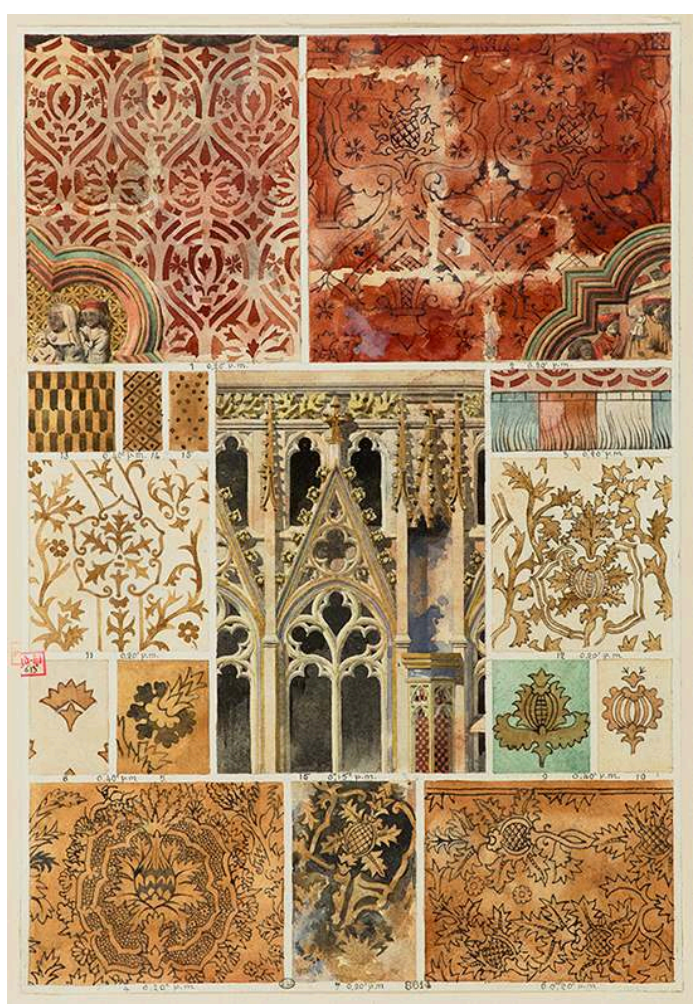

Henri-Louis Lafillée. Clôtures du chœur de la cathédrale d'Amiens. Vers 1890. Aquarelle sur papier. H 15,$5 ; \mathrm{L}: 9,8$. ENSBA, bibliothèque du palais des Études. FNAC 771 (284).

(c) ENSBA. 


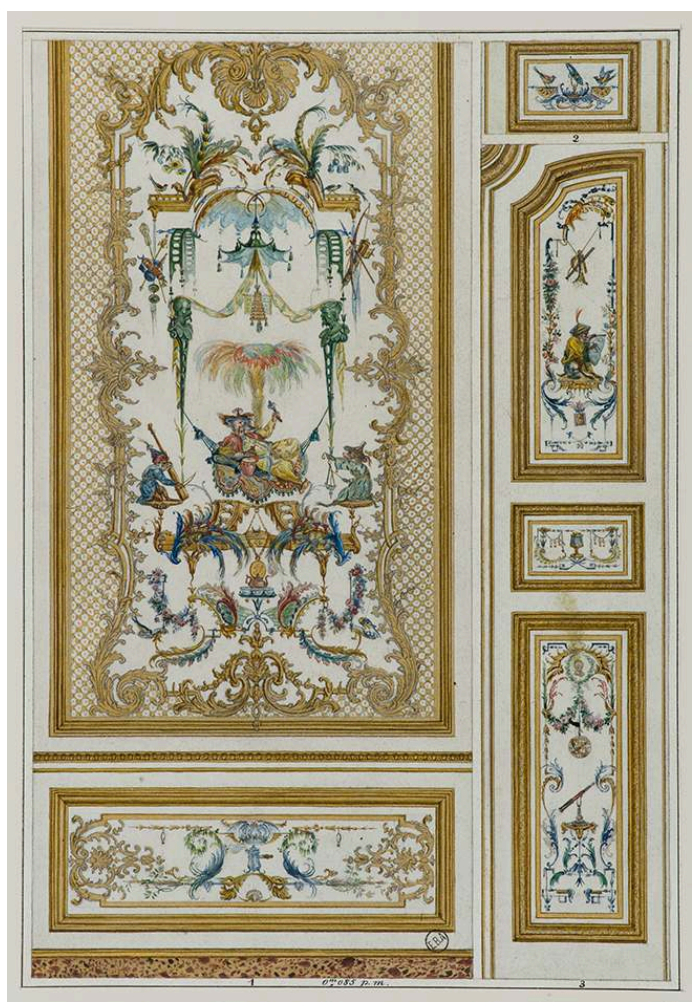

Henri Gélis-Didot. Panneaux du Salon des Singes au château de Chantilly (Oise). Vers 1890. Aquarelle sur papier. H: 31,5 ; L : 21,4. ENSBA, bibliothèque du palais des Études. FNAC 1502 (50).

(c) ENSBA.

Trente ans avant la création de la galerie des peintures par Paul Deschamps (1888-1974) au palais du Trocadéro, l'album de Lafillée et Gélis-Didot offre aux artistes l'opportunité d'approfondir leur connaissance du répertoire stylistique propre aux arts roman, gothique, Renaissance et classique grâce à des exemples empruntés à des monuments aussi divers que l'église de Saint-Savin-sur-Gartempe, la cathédrale d'Amiens (fig. 18), le château de Chantilly (fig. 19) ou des hôtels particuliers parisiens aujourd'hui disparus (fig. 20). Le second, réalisé par Jules Bourgoin lors de ses deux voyages en Égypte, invite le public de l'École à découvrir le système d'ornementation mis au point par les architectes arabes au cours du Moyen Âge tout en reproduisant de nombreux monuments encore inconnus des amateurs (fig. 21). 
Figure 20

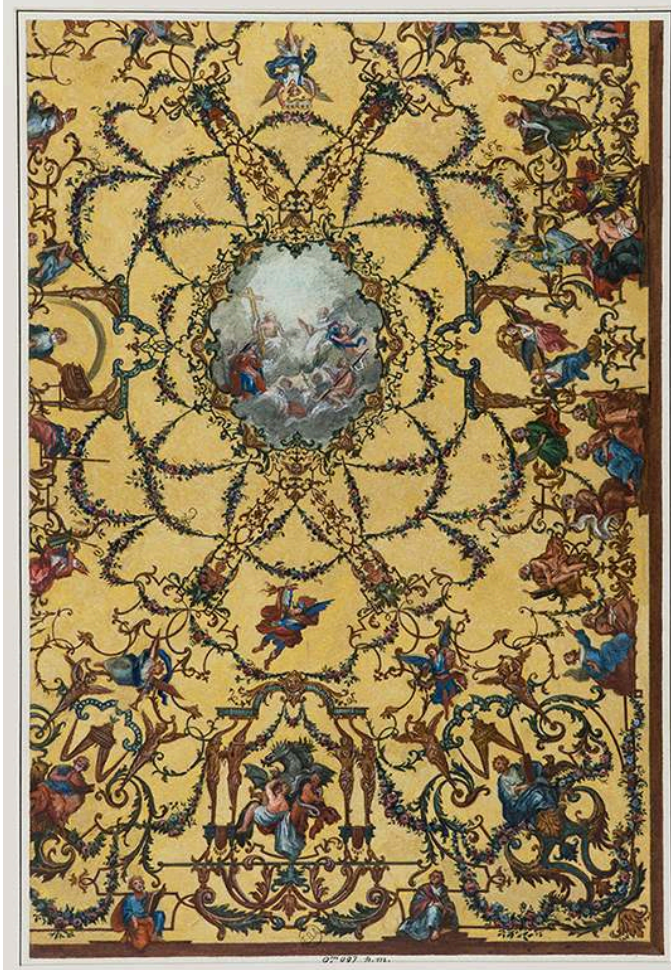

Henri Gélis-Didot. Plafond provenant d'un hôtel situé autrefois rue Picpus à Paris. Vers 1890. Aquarelle sur papier. $\mathrm{H}: 31,2$; L : 23,6. ENSBA, bibliothèque du palais des Études. FNAC 1502 (7).

(c) ENSBA. 


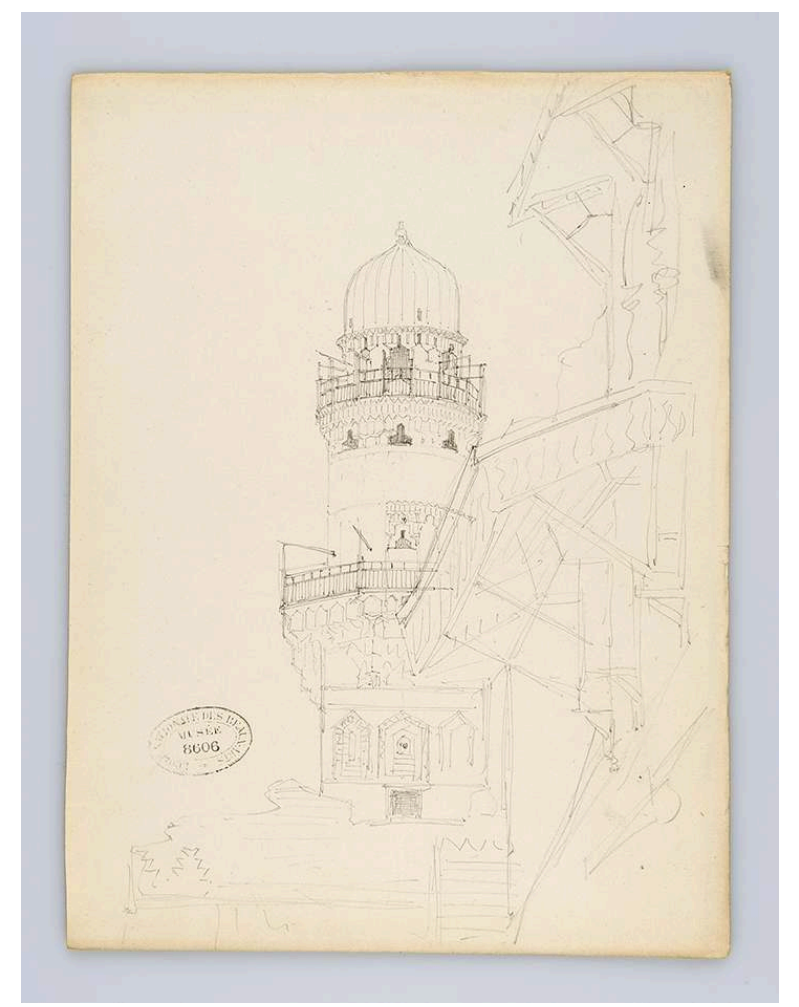

Jules Bourgoin. Minaret du kankah Beibars El Gashankir, rue de Bab-en-Nasr au Caire. Vers 1880. Crayon sur papier. H: 22,2 ; L : 16,7. ENSBA, bibliothèque du palais des Études. FNAC 757 (1).

(c) ENSBA.

De l'Empire à la Troisième République, les dépôts de l'État à l'École des beaux-arts illustrent de manière significative les permanences et les évolutions qui touchent l'enseignement artistique au XIX ${ }^{\mathrm{e}}$ siècle. Dès le début du siècle suivant néanmoins, le déclin progressif de l'intérêt pour la copie, associé à la décision de l'État d'interrompre l'achat régulier des Seconds Grands Prix, entraîne une baisse sensible des attributions au bénéfice de l'établissement. De 1923 à nos jours, 1 dessin, 8 peintures, 2 sculptures, 1 maquette et 1 photographie seulement ont ainsi fait l'objet d'un dépôt dans les locaux de la rue Bonaparte.

En dépit du fait qu'il n'ait pas pris en compte, en raison de leur trop grand nombre, une grande partie des moulages ainsi que les œuvres dites immeubles par nature, le récolement en cours a permis de localiser 4957 œuvres dont certaines n'avaient jamais été inventoriées. C'est le cas en particulier des recueils de dessins d'Alexis Paccard, de Jean-Jacques Clerget, de Théodore Valério ou encore de Claude-Ferdinand Gaillard. Selon la nature des objets, les «taux de redécouverte » demeurent néanmoins très variables. Si le fonds art graphique a révélé la plupart de ses dépôts, à l'exception de quelques séries qui seront probablement découvertes plus tard, il n'en est pas de même pour d'autres catégories d'objets que la disparition de leur valeur d'usage a davantage affectées. Il en est ainsi des maquettes d'architecture, dont une soixantaine seulement parait identifiable depuis leur transfert au musée de Saint-Germain en $1903^{46}$, des grands formats peints réalisés à Rome par les frères Balze ainsi que des envois "plus politiques » comme le portrait de Louis-Philippe par Hippolyte Dominique Holfeld (1804-1872) ou le buste de Napoléon III par Jean-Auguste Barre (1811-1896) ${ }^{47}$. 
41 Même si elles ont aujourd'hui perdu leur fonction pédagogique et éducative, les œuvres retrouvées n'en continuent pas moins à habiter d'une manière extrêmement vivante les locaux de l'École. Dispersées en des lieux très différents, allant des salles de l'espace public aux réserves internes et externes, celles-ci témoignent de l'attention portée par l'ancienne administration des Beaux-Arts à la formation des artistes tout comme à la création contemporaine de notre époque.

\section{NOTES}

1. - Entrepris sous le contrôle de la Commission de récolement des dépôts d'œuvres d'art créée en 1996, ce récolement exclut néanmoins deux catégories d'œuvres : les œuvres classées immeubles par nature telles que les décors pariétaux, traditionnellement financés dans le cas d'un bâtiment propriété de l'État par la direction des Beaux-Arts, ainsi que la plupart des moulages commandés par l'administration qui, en raison de leur trop grand nombre (environ 10000) et de leur dispersion géographique, ne pourraient faire l'objet d'un pointage systématique.

2. - Entre le Premier Empire et la Troisième République, l'administration des Beaux-Arts fut rattachée successivement: au ministère de l'Intérieur, de la Révolution à la chute de la Seconde République (1791-1852), au ministère d'État et à la Maison de l'empereur sous le Second Empire (1853-1870) puis au ministère de l'Instruction publique et des Beaux-Arts, entre 1871 et 1940.

3. - Héritière de l'ancienne Académie de peinture et de sculpture établie au Louvre depuis 1692, l'École dite alors «impériale et spéciale des Beaux-Arts » s'installe à partir de 1807 dans l'enceinte de l'ancien collège des Quatre-Nations, également occupé par l'Institut. Ce n'est qu'en 1829 qu'elle déménage dans les anciens locaux du couvent des Petits-Augustins, rue Bonaparte, où sont édifiés, entre 1824 et 1839, par François Debret et Félix Duban, le bâtiment des loges et le palais des Études puis, entre 1858 et 1862, l'aile du quai Malaquais.

4. - Lettre du ministre de l'Intérieur, Crétet de Champmol, comte d'Empire, au conservateur de la galerie d'architecture, le 4 avril 1809 (Bureau des Beaux-Arts) (Archives nationales, AJ/52/446).

5. - Selon une lettre du ministre de l'Intérieur Camille de Montalivet à Léon Dufourny, directeur de la galerie d'architecture, du 30 janvier 1813 (AN, AJ/52/446), cet ensemble avait été acquis par décret impérial le 14 janvier pour la somme totale de 45910 francs. Composé alors de modèles réalisés en talc, liège et terre cuite d'après les croquis de monuments exécutés par l'architecte au cours de ses voyages, il offrait une collection d'exemplaires uniques tirés des civilisations égyptienne, indienne, persane, grecque, étrusque et romaine. L'ensemble réunissait également des modèles inspirés par l'architecture médiévale comme la tour de Pise.

6. - D'après la lettre du ministre de l'Intérieur Tanneguy Duchâtel au président de l'École royale des beaux-arts le 8 juin 1839 (AN, AJ/52/446), ces cinq monuments furent achetés pour la somme de 1050 francs. L'ensemble, réalisé en 1838 par le sculpteur Fouquet, réunissait un tombeau d'ordre ionique, un petit tombeau lycien à Macri, le tombeau dit d'Amyntas à Macri en Lycie, une porte de la ville d'Assos en Troade dans son état actuel, une poterne de construction grecque voisine de la porte principale d'Assos en Troade.

7. - Cette collection en liège, présentée lors de l'Exposition des produits de l'industrie de 1839, fut acquise le 20 août 1839. Elle comprenait 25 modèles désignés de la manière suivante : «Bains d'Auguste, Amphithéâtre de Nîmes, La tour Magne, état actuel, La tour Magne, restaurée, Porte 
d'Auguste, état actuel, Porte d'Auguste, restaurée, Porte de France, Maison carrée, Pont Ambroise, Pont du Gard, Arc de triomphe d'Orange, Théâtre d'Orange, Cirque d'Orange, Temple de la maison basse, Mausolée, Arc de triomphe de Saint-Rémy, Cénotaphe, Colonne de Riez, Prétoire, Pont Flavien, Arc de triomphe de Carpentras, Obélisque, Colonnes du Forum, Théâtre d'Arles, Amphithéâtre d'Arles. » (AN, AJ/52/446).

8. - « Rapport fait par MM. Dufourny, Durand et Vaudoyer le 19 décembre 1808 au ministre de l'intérieur Crétet de Champmol au sujet de la collection de modèles d'architecture de M. Cassas » (AN, AJ/52/446)

9. - La première campagne se déroula entre 1825 et 1826 , sous la direction de Pierre-Narcisse Guérin (1774-1833), la deuxième fut conduite en Toscane par le peintre Édouard-François Bertin (1797-1871) puis par le conservateur des collections de l'École, Louis Peisse (1803-1880), entre 1834 et 1837, la troisième fut menée sous la direction d'Ingres (1780-1867) entre 1838 et 1841.

10. - Le tableau de Xavier-Alexandre Sigalon fut commandé à l'artiste par décision du 26 novembre 1834, pour la somme de 8000 francs (AN, F/21/613). Il fut installé dans la chapelle des Petits-Augustins en août 1837 et inauguré le mois suivant (STENDHAL. Journal. Dans CEuvres intimes. Éd. Victor Del Litto. Paris: Gallimard, «Bibliothèque de la Pléiade », 1981-1982, t. II, 1818-1842, p. 300).

11. - Les 8 toiles d'après les Chambres étaient: L'École d'Athènes, Le Parnasse, L'Incendie du Bourg, Héliodore chassé du Temple, La Messe de Bolsena, La Délivrance de saint Pierre, La Bataille du Pont-Milvius et L'Allocution aux troupes. L'ensemble de ces œuvres arriva à Paris en 1847 (AN, F/21/5).

12. - Le transfert de ces toiles au palais des Champs-Élysées est mentionné dans la lettre du directeur des Beaux-Arts Charles Blanc au directeur de l'École nationale des beaux-arts Eugène Guillaume le 14 novembre 1872 (AN, AJ/52/445).

13. - Henry Auguste Calixte Serrur demanda à la direction des Beaux-Arts d'exécuter cette œuvre afin de parfaire sa connaissance de la peinture vénitienne (AN, F/21/56). Le tableau, endommagé depuis longtemps, venait de faire l'objet d'une restauration intégrale ; il était exposé dans l'une des salles de l'Académie à Venise.

14. - Décret relatif à la réorganisation administrative et pédagogique de l'École impériale et spéciale des Beaux-Arts, Le Moniteur universel, 15 novembre 1863.

15. - L'enseignement de la peinture ne s'effectuait auparavant que dans des ateliers privés, l'École n'ayant pas de cours spécialement dédiés à cet apprentissage (voir : BONNET, Alain. L'Enseignement des arts au XIXe siècle. La réforme de l'École des beaux-arts de 1863 et la fin du modèle académique. Rennes : PUR, 2006.)

16. - Voir le site : http://cths.fr/an/savant.php?id=831 [consulté le 13/02/2019].

17. - Près de 9000 moulages furent encore déposés à l'École entre 1864 et 1878 . La majorité provenait du fonds réuni par le graveur Alexis-Joseph Depaulis (1792-1867), acquis par la surintendance des Beaux-Arts auprès de sa veuve le 20 décembre 1867 pour 6400 francs (AN, F/ 21/616). L'ensemble se compose de 8517 objets de petite taille (statuettes, bas-reliefs, camées, intailles), issus des horizons géographique et historique les plus divers (Égypte, Grèce, Rome, Moyen Âge occidental et byzantin, Renaissance, époque moderne). L'année suivante, les collections s'enrichissent de 148 autres pièces antiques moulées en Italie sous la direction de Charles Ravaisson-Mollien (1848-1919), membre de l'Académie des inscriptions et belles-lettres.

18. - Une seule maquette fut déposée sous le Second Empire. Il s'agit d'une réduction de la salle des Deux Sœurs de l'Alhambra, acquise le 25 janvier 1866 pour 2000 francs auprès de l'architecte Émile Boeswillwald (1815-1896). Ce dernier la tenait d'un Espagnol du nom de Thomas Pérez qui la lui vendit lors de sa présentation à l'Exposition des beaux-arts appliquée à l'industrie, en 1865 (AN, F/21/120).

19. - Installé au palais des Champs-Élysées, cet établissement réunissait des copies acquises par l'État depuis le début du XIX ${ }^{\mathrm{e}}$ siècle. Il ferma dès 1873, après la démission de son fondateur.

20. - MÜNTZ, Eugène. Guide de l'École nationale des beaux-arts. Paris : 1889, Quantin, p. 160. 
21. - Voir le site : http://cths.fr/an/savant.php?id=1058 [consulté le 13/02/2019].

22. - Entre 1865 et 1923, l'administration des Beaux-Arts procéda à l'achat systématique de tous les Seconds Grands Prix en architecture, peinture, sculpture, gravure et gravure en médaille. Seules les œuvres issues du concours d'architecture, de gravure et dans certains cas, de gravure en médaille, feront l'objet d'un dépôt à l'École, les œuvres des concours de peinture et de sculpture étant destinées à l'embellissement des édifices publics (ministères, préfectures, églises) ou à des musées.

23. - Au cours de leur séjour à la villa Médicis, les Premiers Grands Prix étaient tenus d'envoyer chaque année à l'École les résultats de leurs travaux. Seuls les envois de $4^{\mathrm{e}}$ année, dont le travail, en architecture, était dédié à l'étude et à la restauration d'un édifice antique, devenaient propriété de l'École.

24. - Les 8 dessins de Tétaz, datés entre 1846 et 1847, furent acquis auprès de sa veuve pour la somme de 1000 francs le 14 juillet 1866 (AN, F/21/183). Conçus pour faire partie d'un envoi de $2^{\mathrm{e}}$ année, ils furent exposés au Salon des artistes vivants de 1866 sous le numéro 3070 et retinrent, selon la correspondance de l'administration, l'attention des amateurs. Le lot de dessins fut attribué officiellement à l'École le 29 octobre 1866.

25. - Les 376 dessins préparatoires d'Alexis Paccard sur le Parthénon furent exécutés entre 1842 et 1846 (AN, F/21/169).

26. - Cet ensemble de 461 dessins réalisés lors du séjour de l'artiste à l'Académie de France entre 1836 et 1840 puis en Méditerranée orientale de 1841 à 1843 fut acheté à sa veuve le 14 octobre 1878 pour 1500 francs (AN, F/21/204).

27. - Acquisition des dessins de Valério lors de ses voyages en Hongrie, Croatie et provinces danubiennes. Lettre du directeur des Beaux-Arts à Eugène Guillaume, le 31 mars 1869 (palais des Tuileries) (AN, AJ/52/444.).

28. - Catalogue: TRAPADOUX, Marc. L'Eeuvre de M. Charles Cordier. Galerie anthropologique et ethnologique pour servir à l'histoire des races. Paris : C. Lahure, 1860. (disponible sur Gallica).

29. - Vente Barye. «Liste des œuvres acquises par l'administration des Beaux-Arts lors de la vente des œuvres du sculpteur à l'hôtel Drouot, salles 8 et 9 les lundi 7, mardi 8 , mercredi 9 , jeudi 10 , vendredi 11 et samedi 12 février 1876 par le ministère de $\mathrm{M}^{\mathrm{e}}$ Charles Pillet, commissairepriseur 10 rue de la Grange-Batelière » (AN, F/21/194).

30. - ÉMERIC-DAVID, Toussaint-Bernard. Recherches sur l'art statuaire, considéré chez les anciens et chez les modernes, ou Mémoire sur cette question proposée par l'Institut national de France: Quelles ont été les causes de la perfection de la sculpture antique et quels seroient les moyens d'y atteindre? Paris : Vve Nyon aîné, an XIII-1805.

31. - Lettre du directeur de l'École nationale des beaux-arts Eugène Guillaume à Philippe de Chennevières, directeur des Beaux-Arts, le 19 janvier 1876 (AN, F/21/616).

32. - Les onze copies d'après La Création de Michel-Ange, exécutées par Paul Baudry lors de son séjour à l'Académie de France entre 1865 et 1866 et achetées par la direction des Beaux-Arts le 22 avril 1885, restent l'ensemble le plus spectaculaire de cette période (AN, F/21/2154).

33. - Commandé en 1861 à Auguste-Alexandre Dumont (1801-1884), Premier Grand Prix de Rome et professeur à l'École, sur proposition du secrétaire perpétuel de l'Académie des beaux-arts Michel Halévy, ce buste en marbre fut terminé en 1866 (AN, F/21/136). Réalisé pour honorer la mémoire du fondateur de l'éphémère musée des Monuments français, il fut alors placé dans la travée centrale du premier étage du portique de l'ancien château d'Anet. Il est aujourd'hui conservé à l'intérieur de la chapelle.

34. - Ce buste, sculpté en 1873 par Gabriel Faraill (1839-1892), ancien élève de Jean-Baptiste Farochon, fut commandé par l'administration des Beaux-Arts sur proposition d'Eugène Guillaume (AN, F/21/216).

35. - Sur cet architecte, voir le site: http://cths.fr/an/savant.php?id=107193 [consulté le 13/02/2019]. Réalisé par le sculpteur Jean-Louis Schroeder (1828-1898), ce portrait est la réplique 
en marbre d'un premier buste, alors propriété de la famille Jaÿ, exposé au Salon des artistes vivants de 1873 sous le numéro 1877 (AN, F/21/253).

36. - Voir le site : http://cths.fr/an/savant.php?id=107244 [consulté le 13/02/2019]. Fruit d'une collaboration avec l'architecte Georges-Ernest Coquart, auteur de la colonne-piédestal, l'œuvre fut livrée le 10 février 1886 (AN, F/21/2085).

37. - Voir le site : http://cths.fr/an/savant.php?id=124794 [consulté le 13/02/2019].

38. - La statue d'Henri Chapu fut présentée au Salon des artistes vivants en 1875 sous le numéro 2940. Le monument fut inauguré le 12 août 1876 (AN, F/21/4856).

39. - À ces treize commandes se sont ajoutés deux bustes réalisés précédemment (Jean-BaptisteEugène Farochon par Gabriel Faraill et Alphonse-François Marie Jaÿ par Jean-Louis Désiré Schroeder) ainsi que trois portraits peints : Charles-François Nanteuil par son fils Paul Nanteuil, le sculpteur Henri Chapu par Léon Bonnat et Jules-Élie Delaunay par lui-même.

40. - Commandé le 6 juillet 1895 au fondeur Edmond Gruet, le buste de Charles Garnier d'après l'original de Jean-Baptiste Carpeaux faisait partie d'un lot comprenant 6 autres effigies dont celles d'Henri Delaborde par Justin-Chrysostome Sanson (1833-1910), Claude Dejoux par luimême, Guillaume Guillon Lethière, Jean-Pierre Cortot d'après Louis-Denis Caillouette (1790-1868), Hippolyte Flandrin par Eugène-André Oudiné (1810-1887) et Louis-Pierre Henriquel-Dupont par Jules Cavelier (1814-1894) (AN, F/21/2173).

41. - Le 28 mars 1887 la direction des Beaux-Arts fait l'acquisition de deux portefeuilles de dessins réalisés par Gaillard: les 345 dessins et 13 peintures réalisés par l'artiste lors de son séjour à Pompéi et Naples en 1861 en tant que Premier Prix de Rome et les 317 études exécutées d'après La Cène de Léonard de Vinci en vue de l'exécution d'une planche gravée (AN, F/21/2081).

42. - Les 44 dessins de Delaunay proviennent d'un legs effectué en 1893 par les héritiers de l'artiste au profit de la direction des Beaux-Arts qui les donna ensuite à l'École (AN, AJ/52/448).

43. - Ce recueil fut acquis pour la somme de 6000 francs le 11 février 1893 (AN, F/21/2137/A). Sur Lafillée, voir : http://elec.enc.sorbonne.fr/architectes/298 [consulté le 13/02/2019].

44. - L'acquisition des dessins de Gélis-Didot, pour la somme de 5000 francs, date du 29 mai 1901 (AN, F/21/4213).

45. - Réalisée par l'architecte lors de ses deux voyages en Égypte, le premier entre 1863 et 1866, le second de 1880 à 1884, cette série de dessins, proposée à l'achat par Paul Dubois, a été acquise en deux fois, le 9 août 1892 et le 26 décembre 1892 (AN, F/21/2124).

46. - 19 modèles appartenant à la collection Pelet et 34 autres, appartenant en partie à la collection Cassas, furent transférés à Saint-Germain-en-Laye en 1903 (AN, AJ/52/821).

47. - F/21/63. Ce portrait, destiné à orner la salle des prix, avait été donné à l'École le 27 novembre 1856.

\section{RÉSUMÉS}

Héritier de l'un des services de la division des Beaux-Arts, des Sciences et des Spectacles créée en 1791 pour soutenir la création vivante, le Centre national des arts plastiques a entrepris depuis 1996 un récolement de l'ensemble des œuvres acquises et commandées par l'État depuis la Révolution française. L'opération entreprise à l'École nationale supérieure des beaux-arts (ENSBA), à Paris, a permis d'identifier l'attribution à cet établissement d'un lot de près de 15000 pièces d'une exceptionnelle diversité, principalement destinées à enrichir le «musée des 
études » fondé officiellement le 17 septembre 1834, ainsi que les réserves de la bibliothèque, ouverte en 1863. À partir du dépouillement exhaustif des archives de l'administration des BeauxArts (Archives nationales, série F21) et de l'École nationale des beaux-arts (série AJ 52), les investigations conduites ont montré que la nature de ces dépôts était intimement liée aux évolutions pédagogiques et institutionnelles de l'École, comme l'illustrent la prédominance des maquettes et des moulages au cours de la période néoclassique, l'attribution de copies peintes sous le Second Empire, conséquence logique de la création des ateliers de peinture en 1863, ou encore le dépôt de nombreux dessins à caractère ornemental, destinés à accompagner, sous la Troisième République, la mise en place des nouveaux cours d'art décoratifs.

The Centre national des Arts plastiques, the national centre for the plastic arts, is the heir to a service of fine arts, sciences and theatre first created in 1791 in order to support artistic creation. In 1996, the Centre undertook a retrospective inventory of all the works of art commissioned and purchased by the State since the French Revolution. The part of the inventory carried out at the École nationale supérieure des Beaux-Arts (ENSBA), the Paris fine arts school, permitted the identification of a remarkably diversified collection of about 15,000 items which were deposited at this school, intended principally for the 'study museum', officially founded on 17 September 1834 , or the reserve collections of the library, opened in 1863. By a close examination of the archives left by the fine arts administration and by the administration of the school itself (series F 21 and AJ 52 at the national archives), we were able to show how these deposits were closely related to the pedagogical and institutional evolutions of the school. Illustrations of this conclusion are to be seen in the number of models and plaster casts given to the school during the neo-classical period, the number of copies of paintings during the Second Empire-the logical consequence of the creation of painting ateliers in 1863-or the number of ornamental drawings during the Third Republic, destined to accompany the new courses in the decorative arts.

\section{INDEX}

Keywords : Academy, antique, decorative arts, copy, deposits, teaching, model, neo-classical period, romanticism, orientalism

Mots-clés : Académie, antique, art décoratif, copie, dépôt, enseignement, modèle, néoclassicisme, romantisme, orientalisme

\section{AUTEUR}

\section{STÉPHANE ALLAVENA}

Conservateur du patrimoine, mission de récolement, Centre national des arts plastiques, Pôle collection stephane.allavena@culture.gouv.fr 